\title{
A Binary Decision Tree Abstract Domain Functor
}

\author{
Junjie Chen $^{(凶)}$ and Patrick Cousot \\ New York University, New York, USA \\ junjie@cs.nyu.edu
}

\begin{abstract}
We present an abstract domain functor whose elements are binary decision trees. It is parameterized by decision nodes which are a set of boolean tests appearing in the programs and by a numerical or symbolic abstract domain whose elements are the leaves. We first define the branch condition path abstraction which forms the decision nodes of the binary decision trees. It also provides a new prospective on partitioning the trace semantics of programs as well as separating properties in the leaves. We then discuss our binary decision tree abstract domain functor by giving algorithms for inclusion test, meet and join, transfer functions and extrapolation operators. We think the binary decision tree abstract domain functor may provide a flexible way of adjusting the cost/precision ratio in path-dependent static analysis.
\end{abstract}

\section{Introduction}

In past decades, abstract interpretation [5] has been widely and successfully applied to the static analysis and verification of programs. Abstract domains, one of the key concepts in abstract interpretation, aim at collecting information about the set of all possible values of the program variables. The biggest advantage of using abstract domains instead of logic predicates is that they are fully automatic and can easily scale up. Intervals [4], octagons [14] and polyhedra [6] are the most commonly used numerical abstract domains. These abstract domains are inferring a conjunction of linear constraints to maintain the information of all possible values of program variables and/or the possible relationships between them. The absence of disjunctions may cause rough approximations and produce much less precise results, gradually leading to false alarms or even worse to the complete failure to prove the desired program property.

Let us consider the following example which is modified from the one in [9]:

Work supported in part by NSF grant CNS-1446511.

(C) Springer-Verlag Berlin Heidelberg 2015

S. Blazy and T. Jensen (Eds.): SAS 2015, LNCS 9291, pp. 36-53, 2015.

DOI: $10.1007 / 978-3-662-48288-9 \_3$ 
Example 1. A motivating example.

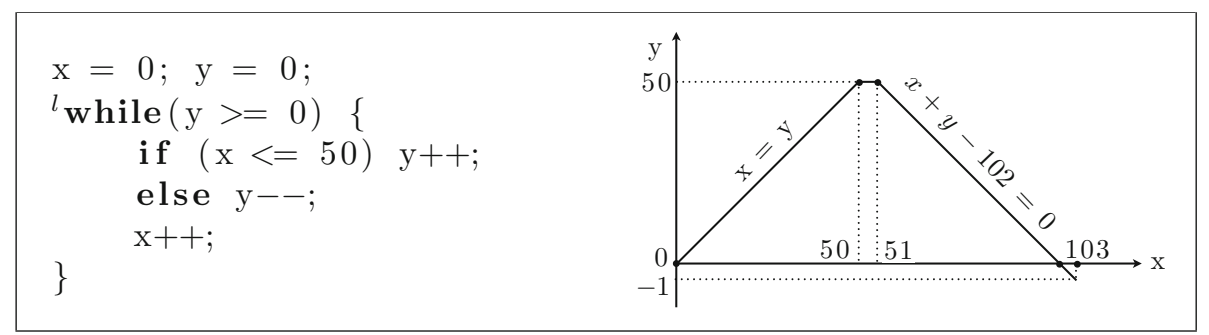

We know that the strongest invariant at program point ${ }^{l}$ is $(0<=\mathrm{x}<=50 \wedge$ $\mathrm{x}=\mathrm{y}) \vee(51<=\mathrm{x}<=103 \wedge x+y-102=0)$. When we use the APRON numerical abstract domain library [12] to generate the invariant at program point $^{l}$, we get $\mathrm{x}>=0 \wedge \mathrm{y}>=-1$ with the box (interval) abstract domain and $\mathrm{y}>=-1 \wedge \mathrm{x}-\mathrm{y}>=0 \wedge \mathrm{x}+52 \mathrm{y}>=0$ with the polka (convex polyhedra) abstract domain. Both analyses are very imprecise compared to the strongest one. This is because the true and false branches of "if $(\mathrm{x}<=50)$ " have different behaviors and those abstract domains do not consider them separately.

Hence, we propose the binary decision tree abstract domain that takes those branches into consideration.

\section{Action Path Semantics}

We consider the following abstract syntax of commands which describes the abstract syntax trees (AST) representing the syntactic structure of source code:

$$
\mathrm{C} \in \mathbb{C}::=\operatorname{skip}|\mathrm{x}=\mathrm{E}| \mathrm{C}_{1} ; \mathrm{C}_{2} \mid \text { if }(\mathrm{B})\left\{\mathrm{C}_{1}\right\} \text { else }\left\{\mathrm{C}_{2}\right\} \mid \text { while }(\mathrm{B})\{\mathrm{C}\}
$$

The trace semantics $\mathcal{S}^{t} \llbracket \mathrm{C} \rrbracket$ of a command $\mathrm{C}$ describes all possible observations of executions of the command C. A trace $\pi$ of length $|\pi| \triangleq n \geq 1$ is a pair $\pi=$ $\langle\bar{\pi}, \underline{\pi}\rangle$ of a finite sequence $\bar{\pi}=\sigma_{0} \sigma_{1} \ldots \sigma_{n-1}$ of states separated by a finite sequence $\underline{\pi}=\mathrm{A}_{0} \mathrm{~A}_{1} \ldots \mathrm{A}_{n-2}$ of actions. States record the current values of variables in the environment/memory as well as a label/control point specifying what remains to be executed while actions record which elementary indivisible elementary program steps are computed during the execution of commands. An action A $\in \mathbb{A}$ is either no operation "skip", an assignment " $\mathrm{x}=\mathrm{E}$ " or a test which output is either true (tt) or false (ff). We use action " $\mathrm{B}$ " to record that the Boolean expression B evaluated to true (tt), while action " $\neg \mathrm{B}$ " records that the Boolean expression B evaluated to false (ff).

The action path abstraction $\alpha^{a}(\mathcal{S})$ collects the set of action paths, that is sequences of actions performed along the traces of a trace semantics $\mathcal{S}$. Given 
a trace $\pi=\langle\bar{\pi}, \underline{\pi}\rangle, \alpha^{a}(\pi) \triangleq \underline{\pi}$ collects the sequence of actions $\underline{\pi}$ executed along that trace, which may be empty $\varepsilon$ for traces reduced to a single state.

Definition 1 (Action Path Abstraction). Given a set of traces $\mathcal{S}$,

$$
\alpha^{a}(\mathcal{S}) \triangleq\left\{\alpha^{a}(\pi) \mid \pi \in \mathcal{S}\right\}
$$

collects the sequences of actions executed along the traces of $\mathcal{S}$.

Note that $\alpha^{a}$ preserves both arbitrary unions and non-empty intersections. We then have the following theorem:

Theorem 1 (Homomorphic Abstraction). Given a function $h: C \mapsto A$, let $\alpha_{h}(X)=\{h(x) \mid x \in X\}$ and $\gamma_{h}(Y)=\{x \mid h(x) \in Y\}$, then $\alpha_{h}$ and $\gamma_{h}$ form a Galois connection:

$$
(\wp(C), \subseteq) \underset{\alpha_{h}}{\stackrel{\gamma_{h}}{\leftrightarrows}}(\wp(A), \subseteq)
$$

Proof. For all $X \in \wp(C)$ and $Y \in \wp(A)$,

$$
\begin{array}{ll} 
& \alpha_{h}(X) \subseteq Y \\
\Longleftrightarrow & \{h(x) \mid x \in X\} \subseteq Y \\
\Longleftrightarrow & \forall x \in X: h(x) \in Y \\
\Longleftrightarrow & X \subseteq\{x \mid h(x) \in Y\} \\
\Longleftrightarrow & X \subseteq \gamma_{h}(Y)
\end{array}
$$

Hence, by defining $\gamma^{a}(\mathcal{A}) \triangleq\left\{\pi \mid \alpha^{a}(\pi) \in \mathcal{A}\right\}$, we will have $\alpha^{a}$ and $\gamma^{a}$ form the Galois connection by Theorem 1 where $h$ is $\alpha^{a}$.

A control flow graph (CFG) is a directed graph, in which nodes correspond to the actions in the program and the edges represent the possible flow of control. The CFG $\mathbb{G} \llbracket \mathrm{C} \rrbracket$ of command $\mathrm{C}$ can be built by structural induction on the syntax of the command $\mathrm{C}$ :

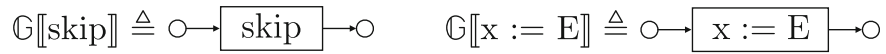

$$
\begin{aligned}
& \mathbb{G} \llbracket \mathrm{C}_{1} ; \mathrm{C}_{2} \rrbracket \triangleq \text { let } \mathbb{G} \llbracket \mathrm{C}_{1} \rrbracket=\circ \longrightarrow \mathrm{C}_{1} \rightarrow \mathrm{O} \text { and } \mathbb{G} \llbracket \mathrm{C}_{2} \rrbracket=\mathrm{O} \longrightarrow \mathrm{C}_{2} \rightarrow \mathrm{O} \text { in } \\
& \mathrm{O} \rightarrow \mathrm{C}_{1} \rightarrow \mathrm{C}_{2} \rightarrow \mathrm{O}
\end{aligned}
$$

$\mathbb{G} \llbracket$ if $(B)\left\{C_{1}\right\}$ else $\left\{\mathrm{C}_{2}\right\} \rrbracket \triangleq$ let $\mathbb{G} \llbracket \mathrm{C}_{1} \rrbracket=0 \rightarrow \mathrm{C}_{1} \rightarrow \mathrm{O}$ and $\mathbb{G} \llbracket \mathrm{C}_{2} \rrbracket=0 \rightarrow \mathrm{C}_{2} \rightarrow \mathrm{O}$ in

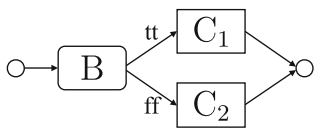

$\mathbb{G} \llbracket$ while $(\mathrm{B})\{\mathrm{C}\} \rrbracket \triangleq$ let $\mathbb{G} \llbracket \mathrm{C} \rrbracket=\mathrm{O} \rightarrow \mathrm{C} \rightarrow \mathrm{O}$ in $\mathrm{O}$ 
Then the action path semantics $\mathcal{G}^{a} \llbracket \mathbb{G} \llbracket \mathrm{C} \rrbracket \rrbracket$ of $\mathrm{CFG} \mathbb{G} \llbracket \mathrm{C} \rrbracket$ of command $\mathrm{C}$ can be defined as:

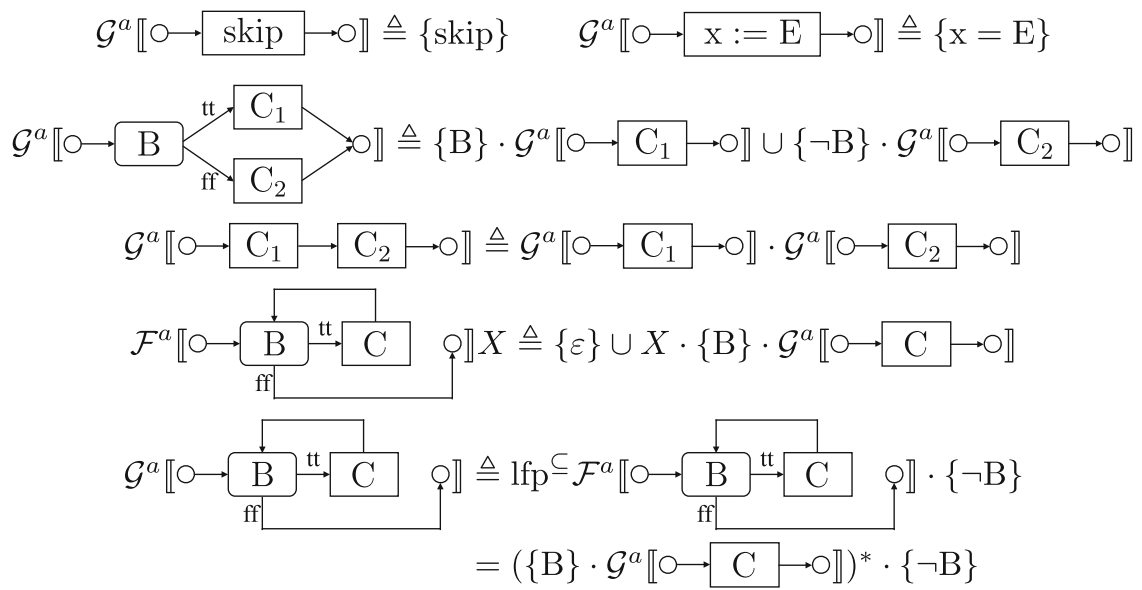

The following Theorem 2 states that the action path semantics of the control flow graph of a program is an over-approximation, hence a sound abstraction, of the action paths that would be collected directly from the trace semantics.

Theorem 2. $\alpha^{a}\left(\mathcal{S}^{t} \llbracket \mathrm{C} \rrbracket\right) \subseteq \mathcal{G}^{a} \llbracket \mathbb{G} \llbracket \mathrm{C} \rrbracket \rrbracket$.

Proof. The proof can be done by the structural induction on the syntax of the command C. More details can be found in the Appendix of [2].

\section{Branch Condition Path Abstraction}

We introduce branch condition graphs $\mathbb{G}^{b} \llbracket \mathrm{C} \rrbracket$ of command $\mathrm{C}$ which can be viewed as further abstractions of the control flow graphs $\mathbb{G} \llbracket \mathrm{C} \rrbracket$. We define the branch condition path semantics $\mathcal{G}^{b} \llbracket \mathbb{G}^{b} \llbracket \mathrm{C} \rrbracket \rrbracket$ as an abstract interpretation $\alpha^{b}$ of the action path semantics $\mathcal{G}^{a} \llbracket \mathbb{G} \llbracket \mathrm{C} \rrbracket \rrbracket$ of the control flow graph $\mathbb{G} \llbracket C \rrbracket$ of command $\mathrm{C}$.

\subsection{Branch Condition Graph}

A branch condition is the test $\mathrm{B}$ occurring in a command "if $(\mathrm{B})\left\{\mathrm{C}_{1}\right\}$ else $\left\{\mathrm{C}_{2}\right\}$ " while a loop condition is the test B occurring in a command "while (B) $\{\mathrm{C}\}$ ". A branch condition graph (BCG) of a program is a directed acyclic graph, in which each node corresponds to a branch condition occurring in the program and has two outgoing edges representing its true and false branches. An edge from node A to node $\mathrm{B}$ means that the branch condition corresponding to node $\mathrm{B}$ occurs after the branch condition corresponding to node $\mathrm{A}$ in the program and there are no other branch conditions occurring between them. A trace from the entry point to the exit point of a BCG is called branch condition path. We use B to denote the true branch while $\neg \mathrm{B}$ denotes the false branch. 
Example 2. Consider the following branch condition graph:

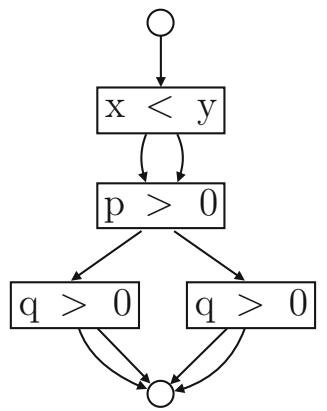

Its branch condition paths include:

$$
\begin{array}{ll}
(\mathrm{x}<\mathrm{y}) \cdot(\mathrm{p}>0) \cdot(\mathrm{q}>0), & \neg(\mathrm{x}<\mathrm{y}) \cdot(\mathrm{p}>0) \cdot(\mathrm{q}>0), \\
(\mathrm{x}<\mathrm{y}) \cdot(\mathrm{p}>0) \cdot \neg(\mathrm{q}>0), & \neg(\mathrm{x}<\mathrm{y}) \cdot(\mathrm{p}>0) \cdot \neg(\mathrm{q}>0), \\
(\mathrm{x}<\mathrm{y}) \cdot \neg(\mathrm{p}>0) \cdot(\mathrm{q}>0), & \neg(\mathrm{x}<\mathrm{y}) \cdot \neg(\mathrm{p}>0) \cdot(\mathrm{q}>0), \\
(\mathrm{x}<\mathrm{y}) \cdot \neg(\mathrm{p}>0) \cdot \neg(\mathrm{q}>0), & \neg(\mathrm{x}<\mathrm{y}) \cdot \neg(\mathrm{p}>0) \cdot \neg(\mathrm{q}>0) .
\end{array}
$$

The branch condition graph $\mathbb{G}^{b} \llbracket \mathrm{C} \rrbracket$, like the $\mathrm{CFG}$, can be defined by structural induction on the syntax of the command $\mathrm{C}$ :

$$
\begin{aligned}
& \mathbb{G}^{b} \llbracket \text { skip } \rrbracket \triangleq \mathrm{O} \longrightarrow \mathrm{O} \quad \mathbb{G}^{b} \llbracket \mathrm{x}:=\mathrm{E} \rrbracket \triangleq \mathrm{O} \longrightarrow \mathrm{O} \\
& \mathbb{G}^{b} \llbracket \mathrm{C}_{1} ; \mathrm{C}_{2} \rrbracket \triangleq \text { let } \mathbb{G}^{b} \llbracket \mathrm{C}_{1} \rrbracket=\mathrm{O} \longrightarrow \mathrm{C}_{1}^{b} \rightarrow \mathrm{O} \text { and } \mathbb{G}^{b} \llbracket \mathrm{C}_{2} \rrbracket=\mathrm{O} \longrightarrow \mathrm{C}_{2}^{b} \rightarrow \mathrm{O} \text { in } \\
& \mathrm{O} \mathrm{C}_{1}^{b} \rightarrow \mathrm{C}_{2}^{b} \rightarrow \mathrm{O}
\end{aligned}
$$

$\mathbb{G}^{b} \llbracket$ if $(\mathrm{B})\left\{\mathrm{C}_{1}\right\}$ else $\left\{\mathrm{C}_{2}\right\} \rrbracket \triangleq$ let $\mathbb{G}^{b} \llbracket \mathrm{C}_{1} \rrbracket=\mathrm{O} \longrightarrow \mathrm{C}_{1}^{b} \rightarrow \mathrm{O}$ and $\mathbb{G}^{b} \llbracket \mathrm{C}_{2} \rrbracket=\mathrm{O} \longrightarrow \mathrm{C}_{2}^{b} \rightarrow \mathrm{O}$

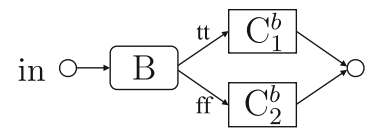

$$
\mathbb{G}^{b} \llbracket \text { while }(\mathrm{B})\{\mathrm{C}\} \rrbracket \triangleq \text { let } \mathbb{G}^{b} \llbracket \mathrm{C} \rrbracket=\circ \longrightarrow \mathrm{C}^{b} \rightarrow \mathrm{O} \text { in } \circ \longrightarrow \mathrm{C}^{b} \rightarrow \circ
$$

Note that the concatenation of $\longrightarrow \mathrm{O}$ and $\mathrm{O} \longrightarrow \mathrm{O}$ is still $\mathrm{O} \longrightarrow \mathrm{O}$

\subsection{Branch Condition Path Abstraction}

We abstract finite action paths $\mathrm{A}_{1} \cdot \mathrm{A}_{2} \cdot \ldots \cdot \mathrm{A}_{n}, n \geq 0$ by the finite branch condition path $\mathrm{A}_{1}^{b} \cdot \mathrm{A}_{2}^{b} \cdot \ldots \cdot \mathrm{A}_{m}^{b}, m \leq n$ where $\mathrm{A}_{1}^{b}=\mathrm{A}_{p}, \mathrm{~A}_{2}^{\bar{b}}=\mathrm{A}_{q}, \ldots, \mathrm{A}_{m}^{b}=\mathrm{A}_{r}, 1 \leq p<q<$ $\ldots<r \leq n$ are distinct branch conditions. The branch condition path is empty $\varepsilon$ when there are no branch conditions occurred in the action path. We say that two branch conditions $A_{1}^{b}, A_{2}^{b}$ are equal if and only if $A_{1}^{b}$ and $A_{2}^{b}$ occur at the same program point. Moreover, each branch condition in the branch condition 
path must be the last occurrence in the action path being abstracted, that is, if $\mathrm{A}_{i}^{b}$ is a branch condition in the branch condition path $\mathrm{A}_{1}^{b} \cdot \mathrm{A}_{2}^{b} \cdot \ldots \cdot \mathrm{A}_{m}^{b}$ abstracting the action path $\mathrm{A}_{1} \cdot \mathrm{A}_{2} \cdot \ldots \cdot \mathrm{A}_{n}$ where $\mathrm{A}_{i}^{b}=\mathrm{A}_{j}$, then $\forall k: j<k \leq n, \mathrm{~A}_{i}^{b} \neq \mathrm{A}_{k}$. Note that we only consider finite action paths hence safety properties.

Condition Path Abstraction. The condition path abstraction collects the set of finite sequences of conditions performed along the action path $\underline{\pi}$ and ignores any skip and assignment in $\underline{\pi}$. Given an action path $\underline{\pi}, \alpha^{c}(\underline{\pi})$ collects the sequence of conditions in the action path, which may be empty $\varepsilon$ when there are no conditions occurred in the action path, by the following induction rules:

$$
\begin{array}{rlrl}
\left.\alpha^{c} \text { (skip }\right) & \triangleq \varepsilon & \alpha^{c}(\mathrm{~B}) & \triangleq \mathrm{B} \\
\alpha^{c}(\mathrm{x}=\mathrm{E}) & \triangleq \varepsilon & \alpha^{c}(\neg \mathrm{B}) \triangleq \neg \mathrm{B} \\
\alpha^{c}\left(\underline{\pi}_{1} \cdot \underline{\pi}_{2}\right) & \triangleq \alpha^{c}\left(\underline{\pi}_{1}\right) \cdot \alpha^{c}\left(\underline{\pi}_{2}\right) & &
\end{array}
$$

Note that $\varepsilon \cdot \underline{\pi_{c}}=\underline{\pi_{c}} \cdot \varepsilon=\underline{\pi}_{c}$. Let $\mathbb{A}^{C}$ be the set of conditions and $\left(\mathbb{A}^{C}\right)^{*}$ be the set of finite, possible empty, condition paths. Given a set of action paths $\mathcal{A}$, $\alpha^{c}(\mathcal{A})$ collects the sequences of conditions in the action paths $\mathcal{A}$ :

$$
\begin{aligned}
\alpha^{c} & \in \wp\left(\mathbb{A}^{*}\right) \mapsto \wp\left(\left(\mathbb{A}^{C}\right)^{*}\right) \\
\alpha^{c}(\mathcal{A}) & \triangleq\left\{\alpha^{c}(\underline{\pi}) \mid \underline{\pi} \in \mathcal{A}\right\}
\end{aligned}
$$

It follows that $\alpha^{c}$ preserves arbitrary unions and non-empty intersections. By defining $\gamma^{c}(\mathcal{C}) \triangleq\left\{\underline{\pi} \mid \alpha^{c}(\underline{\pi}) \in \mathcal{C}\right\}$, we will have:

\section{Corollary 1.}

$$
\left(\wp\left(\mathbb{A}^{*}\right), \subseteq\right) \frac{\gamma^{c}}{\alpha^{c}}\left(\wp\left(\left(\mathbb{A}^{C}\right)^{*}\right), \subseteq\right)
$$

Proof. By Theorem 1 where $h$ is $\alpha^{c}$.

Loop Condition Elimination. Given a finite condition path $\underline{\pi_{c}}, \alpha^{d}\left(\underline{\pi_{c}}\right)$ collects the finite sequence of branch conditions (with duplications) by eliminating all loop conditions in $\underline{\pi_{c}}$. This sequence may be empty $\varepsilon$ when there are no branch conditions occurred in $\underline{\pi_{c}}$. Let $\mathbb{A}^{B}$ be the set of branch conditions and $\mathbb{A}^{L}$ be the set of loop conditions, thus $\mathbb{A}^{C} \triangleq \mathbb{A}^{B} \cup \mathbb{A}^{L}$ and $\mathbb{A}^{B} \cap \mathbb{A}^{L}=\emptyset$. Note that we distinguish those conditions by the program points where they occur, not by themselves.

For all $\mathrm{A}^{b} \in \mathbb{A}^{B}$ and $\mathrm{A}^{l} \in \mathbb{A}^{L}$, we have

$$
\alpha^{d}\left(\mathrm{~A}^{b}\right) \triangleq \mathrm{A}^{b} \quad \text { and } \quad \alpha^{d}\left(\mathrm{~A}^{l}\right) \triangleq \varepsilon .
$$

Then given two condition paths $\underline{\pi_{c_{1}}}$ and $\underline{\pi_{c_{2}}}$, we have

$$
\alpha^{d}\left(\underline{\pi_{c_{1}}} \cdot \underline{\pi_{c_{2}}}\right) \triangleq \alpha^{d}\left(\underline{\pi_{c_{1}}}\right) \cdot \alpha^{d}\left(\underline{\pi_{c_{2}}}\right) .
$$


Note that $\varepsilon \cdot \pi_{d}=\pi_{d} \cdot \varepsilon=\pi_{d}$. Let $\left(\mathbb{A}^{B}\right)^{*}$ be the set of finite, possible empty, sequences of branch conditions. Given a set of condition paths $\mathcal{C}, \alpha^{d}(\mathcal{C})$ collects the sequences of branch conditions (with duplications) from the condition paths $\mathcal{C}$ :

$$
\begin{aligned}
\alpha^{d} & \in \wp\left(\left(\mathbb{A}^{C}\right)^{*}\right) \mapsto \wp\left(\left(\mathbb{A}^{B}\right)^{*}\right) \\
\alpha^{d}(\mathcal{C}) & \triangleq\left\{\alpha^{d}\left(\underline{\pi_{c}}\right) \mid \underline{\pi_{c}} \in \mathcal{C}\right\}
\end{aligned}
$$

It's easy to see that $\alpha^{d}$ preserves both arbitrary unions and non-empty intersections. By defining $\gamma^{d}(\mathcal{D}) \triangleq\left\{\underline{\pi_{c}} \mid \alpha^{d}\left(\underline{\pi_{c}}\right) \in \mathcal{D}\right\}$, we will have:

\section{Corollary 2.}

$$
\left(\wp\left(\left(\mathbb{A}^{C}\right)^{*}\right), \subseteq\right) \underset{\alpha^{d}}{\stackrel{\gamma^{d}}{\leftrightarrows}}\left(\wp\left(\left(\mathbb{A}^{B}\right)^{*}\right), \subseteq\right)
$$

Proof. By Theorem 1 where $h$ is $\alpha^{d}$.

Duplication Elimination. The branch condition paths are the sequences of branch conditions without duplications. In this part, we introduce the abstraction function that eliminates duplications in any sequence of branch conditions.

We first define two functions that are used in the abstraction function $\alpha^{\ell}$. Given a sequence $s e q$ and an element $d$ of $s e q$, erase $(s e q, d)$ eliminates all elements in seq that is equal to $d$ :

$$
\begin{array}{r}
\operatorname{erase}\left(d_{1} d_{2} d_{3} \ldots d_{n}, d\right) \triangleq \text { if } d_{1}=d \text { then } \operatorname{erase}\left(d_{2} d_{3} \ldots d_{n}, d\right) \\
\text { else } d_{1} \cdot \operatorname{erase}\left(d_{2} d_{3} \ldots d_{n}, d\right)
\end{array}
$$

Note that erase $(s e q, d)$ may return the empty sequence $\varepsilon$. Then fold(seq) eliminates the duplications of each element in seq starting from the last element:

$$
\begin{aligned}
\operatorname{fold}\left(d_{1} d_{2} \ldots d_{n}\right) \triangleq & \text { if } d_{1} d_{2} \ldots d_{n}=\varepsilon \text { then } \varepsilon \\
& \text { else fold }\left(\operatorname{erase}\left(d_{1} d_{2} \ldots d_{n-1}, d_{n}\right)\right) \cdot d_{n}
\end{aligned}
$$

Hence, given a sequence of branch conditions $\underline{\pi_{d}}, \alpha^{\ell}\left(\underline{\pi_{d}}\right)=f o l d\left(\underline{\pi_{d}}\right)$ eliminates duplications of each branch condition while keeping its last occurrence in $\pi_{d}$. Let $\mathbb{D}$ be the set of sequences of branch conditions that have duplications. Given a set of sequences of branch conditions $\mathcal{D}, \alpha^{\ell}(\mathcal{D})$ collects branch condition paths (sequences of branch conditions without duplications):

$$
\begin{aligned}
\alpha^{\ell} & \in \wp\left(\left(\mathbb{A}^{B}\right)^{*}\right) \mapsto \wp\left(\left(\mathbb{A}^{B}\right)^{*} \backslash \mathbb{D}\right) \\
\alpha^{\ell}(\mathcal{D}) & \triangleq\left\{\alpha^{\ell}\left(\underline{\pi_{d}}\right) \mid \underline{\pi_{d}} \in \mathcal{D}\right\}
\end{aligned}
$$

Similarly, we have $\alpha^{\ell}$ preserves both arbitrary unions and non-empty intersections. By defining $\gamma^{\ell}(\mathcal{B}) \triangleq\left\{\underline{\pi_{d}} \mid \alpha^{\ell}\left(\underline{\pi_{d}}\right) \in \mathcal{B}\right\}$, we will have:

\section{Corollary 3.}

$$
\left(\wp\left(\left(\mathbb{A}^{B}\right)^{*}\right), \subseteq\right) \frac{\gamma^{\ell}}{\alpha^{\ell}}\left(\wp\left(\left(\mathbb{A}^{B}\right)^{*} \backslash \mathbb{D}\right), \subseteq\right)
$$

Proof. By Theorem 1 where $h$ is $\alpha^{\ell}$. 
Branch Condition Path Abstraction. The branch condition path abstraction $\alpha^{b} \llbracket \mathcal{A} \rrbracket$ collects the branch condition paths, which is the set of sequences of branch conditions with no duplications along the action paths in $\mathcal{A}$. It can be defined by the composition of $\alpha^{c}, \alpha^{d}, \alpha^{\ell}$ defined in the previous sections as:

$$
\begin{aligned}
\alpha^{b} & \in \wp\left(\mathbb{A}^{*}\right) \mapsto \wp\left(\left(\mathbb{A}^{B}\right)^{*} \backslash \mathbb{D}\right) \\
\alpha^{b}(\mathcal{A}) & \triangleq \alpha^{\ell} \circ \alpha^{d} \circ \alpha^{c}(\mathcal{A})
\end{aligned}
$$

Respectively, the concretization function $\gamma^{b}(\mathcal{B})$ can be defined by the composition of $\gamma^{c}, \gamma^{d}, \gamma^{\ell}$ as:

$$
\begin{aligned}
\gamma^{b} & \in \wp\left(\left(\mathbb{A}^{B}\right)^{*} \backslash \mathbb{D}\right) \mapsto \wp\left(\mathbb{A}^{*}\right) \\
\gamma^{b}(\mathcal{B}) & \triangleq \gamma^{c} \circ \gamma^{d} \circ \gamma^{\ell}(\mathcal{B})
\end{aligned}
$$

It follows that $\alpha^{b}$ and $\gamma^{b}$ form a Galois connection:

$$
\left(\wp\left(\mathbb{A}^{*}\right), \subseteq\right) \underset{\alpha^{b}}{\stackrel{\gamma^{b}}{\leftrightarrows}}\left(\wp\left(\left(\mathbb{A}^{B}\right)^{*} \backslash \mathbb{D}\right), \subseteq\right)
$$

Proof. The composition of Galois connections is still a Galois connection.

Example 3. In Example 1, let $\mathcal{A}$ be all possible action path semantics of its CFG, then $\alpha^{b}(\mathcal{A})=\{\mathrm{x} \leq 50, \neg(\mathrm{x} \leq 50)\}$.

\section{Binary Decision Tree Abstract Domain Functor}

We introduce the binary decision tree abstract domain functor to represent and manipulate invariants in the form of binary decision trees. The abstract property will be represented by the disjunction of leaves which are separated by the values of binary decisions, i.e., boolean tests, which will be organized in the decision nodes of the binary decision trees.

\subsection{Definition}

Given the trace semantics $\mathcal{S}^{t} \llbracket \mathrm{P} \rrbracket$ of a program $\mathrm{P}, \alpha^{b} \circ \alpha^{a}\left(\mathcal{S}^{t} \llbracket \mathrm{P} \rrbracket\right)$ abstracts $\mathcal{S}^{t} \llbracket \mathrm{P} \rrbracket$ into a finite set $\mathcal{B}$ of branch condition paths where $\mathcal{B}=\left\{\underline{\pi}_{b}, \ldots, \underline{\pi}_{b}\right\}$. Then for $\underline{\pi}_{i} \in \mathcal{B}$, we have $\gamma^{a} \circ \gamma^{b}\left(\underline{\pi}_{b}\right) \cap \mathcal{S}^{t} \llbracket \mathrm{P} \rrbracket \subseteq \mathcal{S}^{t} \llbracket \mathrm{P} \rrbracket$ and $\bar{\bigcup}_{i \leq N}\left(\gamma^{a} \circ \gamma^{b}\left(\underline{\pi}_{b}\right) \cap\right.$ $\left.\mathcal{S}^{t} \llbracket \overline{\mathrm{P} \rrbracket}\right)=\mathcal{S}^{t} \llbracket \mathrm{P} \rrbracket$. Moreover, for all distinct pairs $\left(\underline{\pi}_{b}, \underline{\pi}_{b_{2}}\right) \in \mathcal{B} \times \mathcal{B}$, we have $\left(\gamma^{a} \circ \gamma^{b}\left(\underline{\pi}_{b}\right) \cap \mathcal{S}^{t} \llbracket \mathrm{P} \rrbracket\right) \cap\left(\gamma^{a} \circ \gamma^{b}\left(\underline{\pi}_{b}\right) \cap \mathcal{S}^{t} \llbracket \mathrm{P} \rrbracket\right)=\emptyset$. Each branch condition path $\underline{\pi}_{b}$ defines a subset of the trace semantics $\mathcal{S}^{t} \llbracket \mathrm{P} \rrbracket$ of a program P. If we can

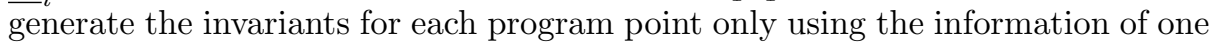
subset of the trace semantics, then for each program point, we will get a finite set of invariants. It follows that the disjunction of such set of invariants forms the invariant of that program point. Hence, we encapsulate the set of branch condition paths into the decision nodes of a binary decision tree where each 
top-down path (without leaf) of the binary decision tree represents a branch condition path, and store in each leaf nodes the invariant generated from the information of the subset of the trace semantics defined by the corresponding branch condition path.

We denote the binary decision tree in the parenthesized form

$$
\llbracket \mathrm{B}_{1}: \llbracket \mathrm{B}_{2}:\left(P_{1} \mid\right),\left(\left|P_{2}\right|\right) \rrbracket, \llbracket \mathrm{B}_{3}:\left(\left|P_{3}\right|\right),\left(\left|P_{4}\right|\right) \rrbracket \rrbracket
$$

where $\mathrm{B}_{1}, \mathrm{~B}_{2}, \mathrm{~B}_{3}$ are decisions (branch conditions) and $P_{1}, P_{2}, P_{3}, P_{4}$ are invariants. It encodes the fact that either if $\mathrm{B}_{1}$ and $\mathrm{B}_{2}$ are both true then $P_{1}$ holds, or if $\mathrm{B}_{1}$ is true and $\mathrm{B}_{2}$ is false then $P_{2}$ holds, or if $\mathrm{B}_{1}$ is false and $\mathrm{B}_{3}$ is true then $P_{3}$ holds, or if $\mathrm{B}_{1}$ and $\mathrm{B}_{3}$ are both false then $P_{4}$ holds. The parenthesized representation of binary trees uses $(\ldots)$ for leaves and $\llbracket \mathrm{B}: t_{l}, t_{r} \rrbracket$ for the decision $\mathrm{B}$ and $t_{l}$ (resp. $t_{r}$ ) represents its left subtree (resp. right subtree). In first order logic, the above binary decision tree would be be written as $\left(\mathrm{B}_{1} \wedge \mathrm{B}_{2} \wedge P_{1}\right) \vee\left(\mathrm{B}_{1} \wedge \neg \mathrm{B}_{2} \wedge P_{2}\right) \vee\left(\neg \mathrm{B}_{1} \wedge \mathrm{B}_{3} \wedge P_{3}\right) \vee\left(\neg \mathrm{B}_{1} \wedge \neg \mathrm{B}_{3} \wedge P_{4}\right)$ with an implicit universal quantification over free variables.

Let $D(\mathcal{B})$ denote the set of all branch conditions appearing in $\mathcal{B}$. Let $\beta=$ $\mathrm{B}$ or $\neg \mathrm{B}$ and $\mathcal{B} \backslash \beta$ denote the removal of $\beta$ and all branch conditions appearing before in each branch condition path in $\mathcal{B}$, then we define the binary decision tree as:

Definition 2. A binary decision tree $t \in \mathbb{T}\left(\mathcal{B}, \mathbb{D}_{\ell}\right)$ over the set $\mathcal{B}$ of branch condition paths (with concretization $\gamma^{a} \circ \gamma^{b}$ ) and the leaf abstract domain $\mathbb{D}_{\ell}$ (with concretization $\gamma_{\ell}$ ) is either $(p \mid)$ with $p$ is an element of $\mathbb{D}_{\ell}$ and $\mathcal{B}$ is empty or $\llbracket B: t_{t}, t_{f} \rrbracket$ where $B \in D(\mathcal{B})$ is the first element of all branch condition paths $\pi_{b} \in \mathcal{B}$ and $\left(t_{t}, t_{f}\right)$ are the left and right subtree of $t$ represent its true and false branch such that $t_{t}, t_{f} \in \mathbb{T}\left(\mathcal{B}_{\backslash \beta}, \mathbb{D}_{\ell}\right)$.

Example 4. In Example 1, the binary decision tree at program point ${ }^{l}$ will be $t=\llbracket \mathrm{x} \leq 50:(0 \leq \mathrm{x} \leq 50 \wedge \mathrm{x}=\mathrm{y} \mid),(51 \leq \mathrm{x} \leq 103 \wedge \mathrm{x}+\mathrm{y}-102=0) \rrbracket$.

Let $\rho$ be the concrete environment assigning concrete values $\rho(x)$ to variables $x$ and $\llbracket e \rrbracket \rho$ for the concrete value of the expression $e$ in the concrete environment $\rho$, we can then define the concretization of the binary decision tree as

Definition 3. The concretization of a binary decesion tree $\gamma_{t}$ is either

$$
\gamma_{t}((|p|)) \triangleq \gamma_{\ell}(p)
$$

when the binary decision tree is reduced to a leaf or

$$
\begin{aligned}
\gamma_{t}\left(\llbracket B: t_{t}, t_{f} \rrbracket\right) \triangleq\{\rho \mid \llbracket B \rrbracket \rho & =\text { true } \Longrightarrow \rho \in \gamma_{t}\left(t_{t}\right) \wedge \\
\llbracket B \rrbracket \rho & \left.=\text { false } \Longrightarrow \rho \in \gamma_{t}\left(t_{f}\right)\right\}
\end{aligned}
$$

when the binary decision tree is rooted at a decision node.

Given $t_{1}, t_{2} \in \mathbb{T}\left(\mathcal{B}, \mathbb{D}_{\ell}\right)$, we say that $t_{1} \equiv_{t} t_{2}$ if and only if $\gamma_{t}\left(t_{1}\right)=\gamma_{t}\left(t_{2}\right)$. Let $\mathbb{T}\left(\mathcal{B}, \mathbb{D}_{\ell}\right) \backslash \equiv_{t}$ be the quotient by the equivalence relation $\equiv_{t}$. The binary decision tree abstract domain functor is defined as: 
Definition 4. A binary decision tree abstract domain functor is a tuple

$$
\left\langle\mathbb{T}\left(\mathcal{B}, \mathbb{D}_{\ell}\right) \bigvee_{E_{t}}, \sqsubseteq_{t}, \perp_{t}, \top_{t}, \sqcup_{t}, \sqcap_{t}, \nabla_{t}, \Delta_{t}\right\rangle
$$

on two parameters, a set $\mathcal{B}$ of branch condition paths and a leaf abstract domain $\mathbb{D}_{\ell}$ where $\left(\mathbb{T}\right.$ is short for $\left.\mathbb{T}\left(\mathcal{B}, \mathbb{D}_{\ell}\right) \backslash \equiv_{t}\right)$

$$
\begin{aligned}
& P, Q, \ldots \in \mathbb{T} \\
& \sqsubseteq_{t} \in \mathbb{T} \times \mathbb{T} \rightarrow\{\text { false, true }\} \\
& \perp_{t}, \top_{t} \in \mathbb{T} \\
& \sqcup_{t}, \sqcap_{t} \in \mathbb{T} \times \mathbb{T} \rightarrow \mathbb{T} \\
& \nabla_{t}, \Delta_{t} \in \mathbb{T} \times \mathbb{T} \rightarrow \mathbb{T}
\end{aligned}
$$

\author{
abstract properties \\ abstract partial order \\ infimum, supremum \\ $\left(\forall P \in \mathbb{T}: \perp_{t} \sqsubseteq_{t} P \sqsubseteq_{t} \top_{t}\right)$ \\ abstract join, meet \\ abstract widening, narrowing
}

The set $\mathcal{B}$ of branch condition paths can be constructed from the CFG of the program. It can be done either in the pre-analysis or on the fly during the analysis. The static analyzer designer should allow to change the maximal length of branch condition paths in $\mathcal{B}$ so as to be able to adjust the cost/precision ratio of the analysis. The leaf abstract domain $\mathbb{D}_{\ell}$ for the leaves could be any numerical or symbolic abstract domains such as intervals, octagons and polyhedra, array domains, etc., or even the reduced product of two or more abstract domains. A list of available abstract domains that can be used at the leaves would be another option of the static analyzer designer. We can use any of these options to build a particular instance of the binary decision tree abstract functor. The advantage of this modular approach is that we can change those options to adjust the cost/precision ratio without having to change the structure of the analyzer.

\title{
4.2 Binary Operations
}

Inclusion and Equality. Given two binary decision tree $t_{1}, t_{2} \in \mathbb{T}\left(\mathcal{B}, \mathbb{D}_{\ell}\right) \backslash$ $\left\{\perp_{t}, \top_{t}\right\}$, we can check $t_{1} \sqsubseteq_{t} t_{2}$ by comparing each pair $\left(\ell_{1}, \ell_{2}\right)$ of leaves in $\left(t_{1}, t_{2}\right)$ where $\ell_{1}$ and $\ell_{2}$ are defined by the same branch condition path $\pi_{b} \in \mathcal{B}$. If each pair $\left(\ell_{1}, \ell_{2}\right)$ satisfies $\ell_{1} \sqsubseteq_{\ell} \ell_{2}$, we can conclude that $t_{1} \sqsubseteq_{t} t_{2}$; otherwise, we have $t_{1} \Phi_{t} t_{2}$.

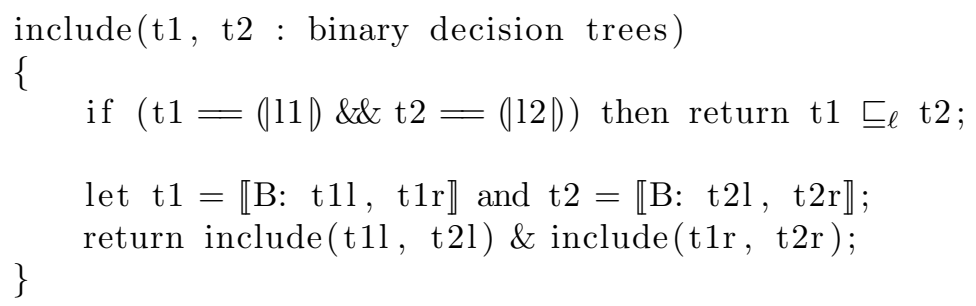


Example 5. We have $\llbracket \mathrm{x} \leq 50:(\mathrm{x}=0 \wedge \mathrm{y}=0),\left(\perp_{\ell}\right) \rrbracket \sqsubseteq \llbracket \mathrm{x} \leq 50:(0 \leq \mathrm{x} \leq$ $1 \wedge \mathrm{x}=\mathrm{y} \mid),\left(\left(\perp_{\ell}\right) \rrbracket\right.$ and $\llbracket \mathrm{x} \leq 50:(\mathrm{x}=0 \wedge \mathrm{y}=0),\left(\perp_{\ell} \mid\right) \rrbracket \nsubseteq \llbracket \mathrm{x} \leq 50:(\mathrm{x}=$ $1 \wedge \mathrm{y}=1 \mid),\left(\perp_{\ell} \mid\right) \rrbracket$.

The equality of $t_{1}$ and $t_{2}$ can be tested by the fact $t_{1}={ }_{t} t_{2} \triangleq t_{1} \sqsubseteq_{t} t_{2} \wedge t_{2} \sqsubseteq_{t}$ $t_{1}$. When the leaf abstract domain $\mathbb{D}_{\ell}$ has $=_{\ell}$, we can also check the equality for each pair $\left(\ell_{1}, \ell_{2}\right)$ of leaves in $\left(t_{1}, t_{2}\right)$ where $\ell_{1}$ and $\ell_{2}$ are defined by the same branch condition path $\pi_{b} \in \mathcal{B}$.

Meet and Join. Given two binary decision tree $t_{1}, t_{2} \in \mathbb{T}\left(\mathcal{B}, \mathbb{D}_{\ell}\right)$, the meet $t=t_{1} \Pi_{t} t_{2}$ can be computed using the meet $\Pi_{\ell}$ in the leaf abstract domain $\mathbb{D}_{\ell}$. Let $\ell_{1}, \ell_{2}$ are leaves of $t_{1}, t_{2}$ respectively, where the same branch condition path $\pi_{b} \in \mathcal{B}$ leads to $\ell_{1}$ and $\ell_{2}$, then $\ell=\ell_{1} \Pi_{\ell} \ell_{2}$ is the leaf of $t$ led by the same branch condition path $\underline{\pi}_{b} \in \mathcal{B}$. After computing each leaf $\ell=\ell_{1} \sqcap_{\ell} \ell_{2}$ in $t$, we then get $t=t_{1} \sqcap_{t} t_{2}$.

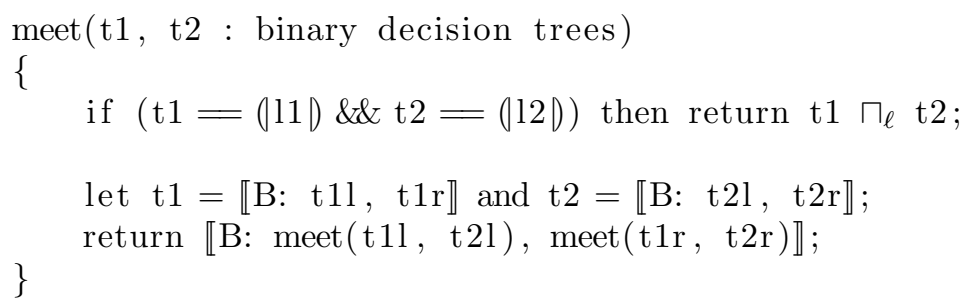

Similar to the meet, we can compute the join $t=t_{1} \sqcup_{t} t_{2}$ using the join $\sqcup_{\ell}$ in the leaf abstract domain $\mathbb{D}_{\ell}$. But instead of computing the join $\ell_{1} \sqcup_{\ell} \ell_{2}$ for each pair $\left(\ell_{1}, \ell_{2}\right)$ of leaves in $\left(t_{1}, t_{2}\right)$ where $\ell_{1}$ and $\ell_{2}$ are led by the same branch condition path $\pi_{b} \in \mathcal{B}$, we also use the branch conditions in $\pi_{b}$ as bound to prevent precision loss. Let $\pi_{b}=\beta_{1} \cdot \beta_{2} \cdot \ldots \cdot \beta_{n}$ where $\beta_{i}=\mathrm{B}_{i}$ or $\overline{\neg \mathrm{B}_{i}}, i=1, \ldots, n$, we have $\ell=\left(\ell_{1} \sqcup_{\ell} \ell_{2}\right) \sqcap_{\ell} \overline{\mathbb{D}}_{\ell}\left(\beta_{1}\right) \sqcap_{\ell} \mathbb{D}_{\ell}\left(\beta_{2}\right) \sqcap_{\ell} \ldots \Pi_{\ell} \mathbb{D}_{\ell}\left(\beta_{n}\right)\left(\mathbb{D}_{\ell}(\beta)\right.$ means the representation of $\beta$ in $\mathbb{D}_{\ell}$, when $\alpha_{\ell}$ exists in the leaf abstract domain $\mathbb{D}_{\ell}$, we can use $\alpha_{\ell}(\beta)$ instead).

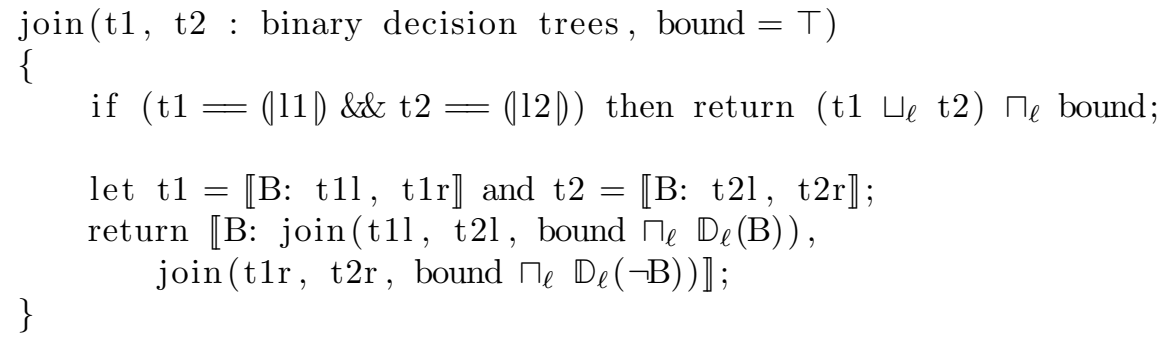


Example 6. Let $t_{1}=\llbracket \mathrm{x} \leq 50:(\mathrm{x}=0 \wedge \mathrm{y}=0),\left(\mid \perp_{\ell}\right) \rrbracket, t_{2}=\llbracket \mathrm{x} \leq 50:(\mid \mathrm{x}=$ $1 \wedge \mathrm{y}=1),\left(\perp_{\ell}\right) \rrbracket, t_{3}=\llbracket \mathrm{x} \leq 50:(0 \leq \mathrm{x} \leq 1 \wedge \mathrm{x}=\mathrm{y}),\left(\perp_{\ell}\right) \rrbracket$, we have $t_{1} \sqcap_{t} t_{2}=\perp_{t}, t_{1} \sqcap_{t} t_{3}=t_{1}$ and $t_{1} \sqcup_{t} t_{2}=t_{3}, t_{2} \sqcap_{t} t_{3}=t_{3}$.

\subsection{Transfer Functions}

We define transfer functions for both tests and assignments. The tests either occur in a loop head or occur in the branch. Hence, we define both loop test transfer function and branch test abstract function for the binary decision tree abstract domain.

Loop Test Transfer Function. The transfer function for the loop tests is simple. Given a binary decision tree $t \in \mathbb{T}\left(\mathcal{B}, \mathbb{D}_{\ell}\right)$ and a loop test $\mathrm{B}$, we first define $t \sqcap_{t} \mathrm{~B}$ as:

$$
\begin{array}{ll}
\perp_{t} \sqcap_{t} \mathrm{~B} & \triangleq \perp_{t} \\
\top_{t} \sqcap_{t} \mathrm{~B} & \triangleq(\mathrm{B}) \\
t \sqcap_{t} \text { false } & \triangleq \perp_{t} \\
t \sqcap_{t} \text { true } & \triangleq t \\
(|p|) \sqcap_{t} \mathrm{~B} & \triangleq\left(p \sqcap_{\ell} \mathbb{D}_{\ell}(\mathrm{B})\right) \\
\llbracket \mathrm{B}^{\prime}: t_{l}, t_{r} \rrbracket \sqcap_{t} \mathrm{~B} \triangleq \llbracket \mathrm{B}^{\prime}: t_{l} \sqcap_{t} \mathbb{D}_{\ell}\left(\mathrm{B}^{\prime} \cap \mathrm{B}\right), t_{r} \sqcap_{t} \mathbb{D}_{\ell}\left(\neg \mathrm{B}^{\prime} \cap \mathrm{B}\right) \rrbracket
\end{array}
$$

Then the transfer function $f_{L} \llbracket \mathrm{B} \rrbracket t$ for the loop test $\mathrm{B}$ of the binary decision tree $t$ can be defined as:

$$
f_{L} \llbracket \mathrm{B} \rrbracket t \triangleq t \sqcap_{t} \mathrm{~B}
$$

Example 7. Let $t$ be the binary decision tree in Example 4 , then $f_{L} \llbracket \mathrm{y}>=0 \rrbracket t=$ $\llbracket \mathrm{x} \leq 50:(0 \leq \mathrm{x} \leq 50 \wedge \mathrm{x}=\mathrm{y}),(51 \leq \mathrm{x} \leq 102 \wedge \mathrm{x}+\mathrm{y}-102=0 \mid) \rrbracket$.

Branch Test Transfer Function. The binary decision tree can be constructed in two different ways. On one hand, it can be generated immediately after the set $\mathcal{B}$ of branch condition paths has been generated in the pre-analysis. In this way, all leaves of the binary decision tree will be set to $T_{\ell}$ for the first program point and $\perp_{\ell}$ for others $\left(T_{\ell}, \perp_{\ell} \in \mathbb{D}_{\ell}\right)$ at the beginning. On the other hand, both binary decision tree and $\mathcal{B}$ can be constructed on the fly during the static analysis. In this last case, we have $\mathcal{B}=\emptyset$ and the binary decision tree $t=\left(\left|\top_{\ell}\right|\right)$ for the first program point and $t=\left(\left|\perp_{\ell}\right|\right)$ for others at the beginning.

In the latter case, the branch test transfer function should first construct the new binary decision tree from the old one by splitting on the branch condition when it has been first met in the analysis. Given a binary decision condition $t \in \mathbb{T}\left(\mathcal{B}, \mathbb{D}_{\ell}\right)$ and a branch test $\mathrm{B}$ that's been first met, there are two situations. One situation is that the branch condition $B$ is independent, that is, it does not occur inside any scope of a branch. In this situation, the new binary decision tree 
$t^{\prime}$ can be constructed by replacing each leaf $p$ in the binary tree $t$ with a subtree $\llbracket \mathrm{B}:\left(\left|p \sqcap_{\ell} \mathbb{D}_{\ell}(\mathrm{B})\right|\right),\left(p \sqcap_{\ell} \mathbb{D}_{\ell}(\neg \mathrm{B}) \mid\right) \rrbracket$. We also have $\mathcal{B}^{\prime}=\left\{\underline{\pi_{b}} \cdot \mathrm{B} \mid \underline{\pi_{b}} \in \mathcal{B}\right\} \cup\left\{\underline{\pi_{b}} \cdot \neg \mathrm{B} \mid\right.$

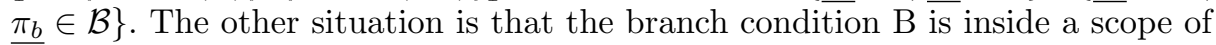
a branch. Let $B$ ' be the condition of the branch and there is no other branch scope between B and B', if B is inside the true branch of B', then the new binary decision tree $t^{\prime}$ can be constructed by replacing each left leaf $p$ of $\mathrm{B}^{\prime}$ in the binary tree $t$ with a subtree $\llbracket \mathrm{B}:\left(\left|p \sqcap_{\ell} \mathbb{D}_{\ell}(\mathrm{B})\right|\right),\left(p \Pi_{\ell} \mathbb{D}_{\ell}(\neg \mathrm{B}) \mid\right) \rrbracket$. We also have $\mathcal{B}^{\prime}=\left\{\underline{\pi_{b}} \cdot \mathrm{B}^{\prime} \cdot \mathrm{B} \mid \underline{\pi_{b}} \cdot \mathrm{B}^{\prime} \in \mathcal{B}\right\} \cup\left\{\underline{\pi_{b}} \cdot \mathrm{B}^{\prime} \cdot \neg \mathrm{B} \mid \underline{\pi_{b}} \cdot \mathrm{B}^{\prime} \in \mathcal{B}\right\} \cup\left(\mathcal{B} \backslash\left\{\underline{\pi_{b}} \cdot \mathrm{B}^{\prime} \mid \underline{\pi_{b}} \cdot \mathrm{B}^{\prime} \in \mathcal{B}\right\}\right)$. If $B$ is inside the false branch of $B^{\prime}$, the right leaves of $B^{\prime}$ instead of left leaves should be replaced by the same subtrees and $\mathcal{B}^{\prime}=\left\{\underline{\pi_{b}} \cdot \neg \mathrm{B}^{\prime} \cdot \mathrm{B} \mid \underline{\pi_{b}} \cdot \neg \mathrm{B}^{\prime} \in\right.$ $\mathcal{B}\} \cup\left\{\underline{\pi_{b}} \cdot \neg \mathrm{B}^{\prime} \cdot \neg \mathrm{B} \mid \underline{\pi_{b}} \cdot \neg \mathrm{B}^{\prime} \in \mathcal{B}\right\} \cup\left(\mathcal{B} \backslash\left\{\underline{\pi_{b}} \cdot \neg \mathrm{B}^{\prime} \mid \underline{\pi_{b}} \cdot \neg \mathrm{B}^{\prime} \in \mathcal{B}\right\}\right)$.

Then in both ways, the branch test transfer function will do the same thing as loop test transfer function. Given the branch test B and the binary decision tree $t \in \mathbb{T}\left(\mathcal{B}, \mathbb{D}_{\ell}\right)$, we have:

$$
f_{B} \llbracket \mathrm{B} \rrbracket t \triangleq t \sqcap_{t} \mathrm{~B}
$$

Example 8. Let $t$ be the binary decision tree in Example 4 , then $f_{B} \llbracket \mathrm{x}<=50 \rrbracket t=$ $\llbracket \mathrm{x} \leq 50:(0 \leq \mathrm{x} \leq 50 \wedge \mathrm{x}=\mathrm{y}),\left(\perp_{\ell}\right) \rrbracket$.

Assignment Transfer Function. Given a binary decision tree $t \in \mathbb{T}\left(\mathcal{B}, \mathbb{D}_{\ell}\right)$, the assignment $\mathrm{x}=\mathrm{E}$ can be performed at each leaf in $t$ by using the assignment transfer function of $\mathbb{D}_{\ell}$. E.g., let $t=\llbracket x \leq 50:(0 \leq x \leq 50),\left(\mid \perp_{\ell}\right) \rrbracket$ and given an assignment $x=x+1$, after performing the assignment transfer function of Polyhedra abstract domain on each leaf of $t$, we will get $t^{\prime}=\llbracket x \leq 50$ : $(1 \leq x \leq 51),\left(\perp_{\ell}\right) \rrbracket$. Generally, the branch condition paths in $\mathcal{B}$ are used as labels separating the abstract properties in disjunctions which are gathered in the leaves. But this is not always the case. For example, in the join operator, we use the branch conditions in $\mathcal{B}$ to reduce the result of the join. After performing the assignment transfer function of leaf abstract domain $\mathbb{D}_{\ell}$ on each leaf, we may also need to manipulate the leaves using the branch condition paths in $\mathcal{B}$. Let's check the above result $t^{\prime}$ after the assignment, it appears that some leaves in the new binary decision tree may not satisfy some branch conditions in the branch condition paths which are leading to them. For example, $1 \leq x \leq 51$ is not satisfying the branch condition $x \leq 50$. We know the violation part is actually satisfying the negation of those branch conditions. Hence we need to use the branch condition $x \leq 50$ to separate $1 \leq x \leq 51$ into $1 \leq x \leq 50 \vee x=51$ and update the corresponding leaves. For example, we have $t^{\prime \prime}=\llbracket x \leq 50$ : $01 \leq x \leq$ 50 ), $(|x=51|) \rrbracket$.

We call this procedure reconstruction on leaves. Given a binary decision tree $t$ after an assignment, we define the procedure as follow:

1. Collecting all leave properties in $t$, let it be $\left\{p_{1}, p_{2}, \ldots, p_{n}\right\}$;

2. For each leaf in $t$, let $\underline{\pi_{b}}=\beta_{1} \cdot \beta_{2} \cdot \ldots \cdot \beta_{n}$ be the branch condition path leading to it. We then calculate $p_{i}^{\prime}=p_{i} \Pi_{\ell}\left(\mathbb{D}_{\ell}\left(\beta_{1} \wedge \beta_{2} \wedge \ldots \wedge \beta_{n}\right)\right)$. 
3. For each leaf in $t$, update it with $p_{1}^{\prime} \sqcup_{\ell} p_{2}^{\prime} \sqcup_{\ell} \ldots \sqcup_{\ell} p_{n}^{\prime}$.

Correctness. Let $p=p_{1} \vee p_{2} \vee \ldots \vee p_{n}$ be the disjunction of all properties in leaves before reconstruction on leaves. For each leaf $\ell_{i}$ in $t$, we have $\ell_{i}=\left(p_{1} \Pi_{\ell}\right.$ $\left.\left(\mathbb{D}_{\ell}\left(\beta_{1}^{i} \wedge \beta_{2}^{i} \wedge \ldots \wedge \beta_{n}^{i}\right)\right)\right) \sqcup_{\ell} \ldots \sqcup_{\ell}\left(p_{n} \Pi_{\ell}\left(\mathbb{D}_{\ell}\left(\beta_{1}^{i} \wedge \beta_{2}^{i} \wedge \ldots \wedge \beta_{n}^{i}\right)\right)\right)=\left(p_{1} \sqcup_{\ell} \ldots \sqcup_{\ell} p_{n}\right) \Pi_{\ell}$ $\left(\mathbb{D}_{\ell}\left(\beta_{1}^{i} \wedge \beta_{2}^{i} \wedge \ldots \wedge \beta_{n}^{i}\right)\right)$ after reconstruction on leaves. We then have the disjunction of all properties in leaves after reconstruction on leaves is $p^{\prime}=\ell_{1} \vee \ldots \vee \ell_{n}=$ $\left(p_{1} \sqcup_{\ell} \ldots \sqcup_{\ell} p_{n}\right) \sqcap_{\ell}\left(\mathbb{D}_{\ell}\left(\beta_{1}^{1} \wedge \beta_{2}^{1} \wedge \ldots \wedge \beta_{n}^{1}\right)\right) \vee \ldots \vee\left(p_{1} \sqcup_{\ell} \ldots \sqcup_{\ell} p_{n}\right) \sqcap_{\ell}\left(\mathbb{D}_{\ell}\left(\beta_{1}^{n} \wedge \beta_{2}^{n} \wedge \ldots \wedge \beta_{n}^{n}\right)\right)=$ $\left(p_{1} \sqcup_{\ell} \ldots \sqcup_{\ell} p_{n}\right) \sqcap_{\ell}\left(\left(\mathbb{D}_{\ell}\left(\beta_{1}^{1} \wedge \beta_{2}^{1} \wedge \ldots \wedge \beta_{n}^{1}\right)\right) \vee \ldots \vee\left(\mathbb{D}_{\ell}\left(\beta_{1}^{n} \wedge \beta_{2}^{n} \wedge \ldots \wedge \beta_{n}^{n}\right)\right)\right)=$ $\left(p_{1} \sqcup_{\ell} \ldots \sqcup_{\ell} p_{n}\right) \sqcap_{\ell}$ true $=p_{1} \sqcup_{\ell} \ldots \sqcup_{\ell} p_{n} \equiv p$. This shows that the reconstruction on leaves procedure will not change the result of the assignment transfer function.

\subsection{Extrapolation Operators}

When the leaf abstract domain $\mathbb{D}_{\ell}$ has strictly increasing and/or strictly decreasing infinite chains, widening and/or narrowing operators are required in the binary decision tree abstract domain to accelerate the convergence of fixpoint iterates.

Widening. Given two binary decision tree $t_{1}, t_{2} \in \mathbb{T}\left(\mathcal{B}, \mathbb{D}_{\ell}\right)$, the widening $t=$ $t_{1} \nabla_{t} t_{2}$ can be computed using the widening $\nabla_{\ell}$ in the leaf abstract domain $\mathbb{D}_{\ell}$ similar to the join operator, that is, computing the widening $\ell_{1} \nabla_{\ell} \ell_{2}$ for each pair $\left(\ell_{1}, \ell_{2}\right)$ of leaves in $\left(t_{1}, t_{2}\right)$ where $\ell_{1}$ and $\ell_{2}$ are led by the same branch condition path $\pi_{b} \in \mathcal{B}$ while the branch conditions in $\pi_{b}$ are also used as the threshold. Let $\overline{\pi_{b}}=\beta_{1} \cdot \beta_{2} \cdot \ldots \cdot \beta_{n}$ where $\beta_{i}=\mathrm{B}_{i}$ or $\neg \overline{\mathrm{B}_{i}}, i=1, \ldots, n$, we have each leaf $\ell=\left(\overline{\ell_{1}} \nabla_{\ell} \ell_{2}\right) \Pi_{\ell} \mathbb{D}_{\ell}\left(\beta_{1}\right) \Pi_{\ell} \mathbb{D}_{\ell}\left(\beta_{2}\right) \Pi_{\ell} \ldots \Pi_{\ell} \mathbb{D}_{\ell}\left(\beta_{n}\right)$.

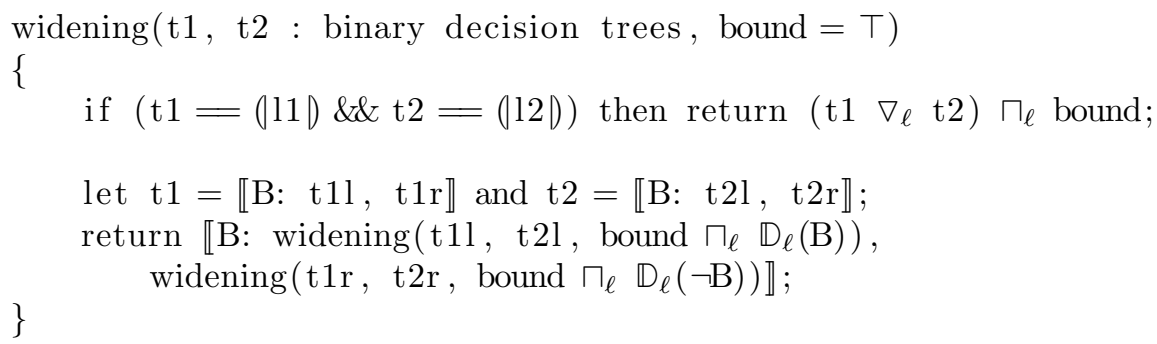

Narrowing. The narrowing operator in the binary decision tree abstract domain is very similar to its meet operator. Given two binary decision tree $t_{1}, t_{2} \in \mathbb{T}\left(\mathcal{B}, \mathbb{D}_{\ell}\right)$, the narrowing $t=t_{1} \Delta_{t} t_{2}$ can be computed using the narrowing $\Delta_{\ell}$ in the leaf abstract domain $\mathbb{D}_{\ell}$. Let $\ell_{1}, \ell_{2}$ are leaves of $t_{1}, t_{2}$ respectively, where the same branch condition path $\underline{\pi_{b}} \in \mathcal{B}$ leads to $\ell_{1}$ and $\ell_{2}$, then $\ell=\ell_{1} \Delta_{\ell} \ell_{2}$ is the leaf of $t$ led by the same branch condition path $\pi_{b} \in \mathcal{B}$. After computing each leaf $\ell=\ell_{1} \Delta_{\ell} \ell_{2}$ in $t$, we then get $t=t_{1} \Delta_{t} t_{2}$. 


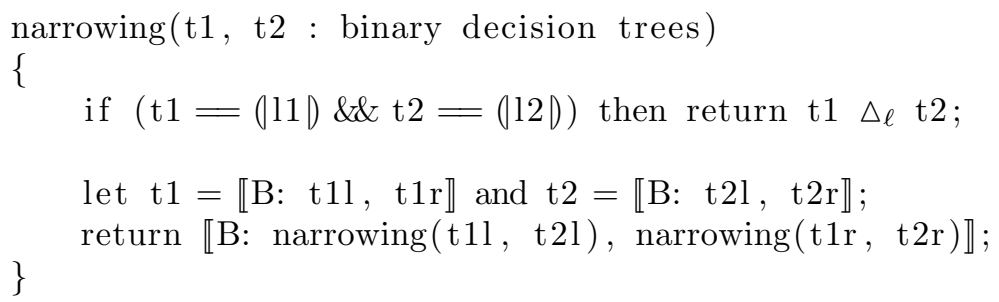

Example 9. Let $t_{1}=\llbracket \mathrm{x} \leq 50:(\mathrm{x}=0 \wedge \mathrm{y}=0 \mid),\left(\perp_{\ell}\right) \rrbracket$ and $t_{2}=\llbracket \mathrm{x} \leq 50$ : $(\mathrm{x}=\mathrm{y} \wedge 0 \leq \mathrm{x} \leq 1 \mid),\left(\perp_{\ell} \mid\right) \rrbracket$. It's easy to see that $t_{1} \subseteq t_{2}$. In polyhedra, we have $(\mathrm{x}=0 \wedge \mathrm{y}=0) \nabla_{t}(\mathrm{x}=\mathrm{y} \wedge 0 \leq \mathrm{x} \leq 1)=\mathrm{x} \geq 0 \wedge \mathrm{x}=\mathrm{y}$. Hence, we have $t_{1} \nabla_{t} t_{2}=\llbracket \mathrm{x} \leq 50:(0 \leq \mathrm{x} \leq 50 \wedge \mathrm{x}=\mathrm{y}),\left(\left(\perp_{\ell}\right) \rrbracket\right.$.

\subsection{Other Operators}

Although the number of branch conditions in a program is always finite, it may still be a very large number. A large number of branch conditions means a large binary decision tree, with a potentially exponential growth which is not acceptable in practice. Hence, we need limit the size (depth) of the binary decision trees.

One method is to eliminate decision nodes by merging their subtrees when the binary decision tree grows too deep. This can be done as follow:

1. Pick up a branch condition $B$. We can simply use the one in the root, or the nearest one to the leaves, or by random. We can also design a ranking function based on the information from the analysis for each branch condition to estimate how likely it is to be eliminated with minimal information loss. Then we always choose the most likely one.

2. Eliminate $B(\mathrm{~B}$ or $\neg \mathrm{B})$ from each branch condition path in $\mathcal{B}$.

3. For each subtree of the form $\llbracket B: t_{t}, t_{f} \rrbracket$, if $t_{t}$ and $t_{f}$ have identical decision nodes, replace it by $t_{t} \sqcup_{t} t_{f}$.

4. Otherwise, there are decision nodes existing only in $t_{t}$ or $t_{f}$. For each of those decision nodes, (recursively) eliminate it by merging its subtrees. When no such decision node exists, we get $t_{t}^{\prime}$ and $t_{f}^{\prime}$, and they must have identical decision nodes, so $\llbracket B: t_{t}, t_{f} \rrbracket$ can be replaced by $t_{t}^{\prime} \sqcup_{t} t_{f}^{\prime}$.

Another method is to generate a smaller $\mathcal{B}$ by abstracting the branch condition paths in $\mathcal{B}$ into shorter ones. We may partition the set of branch conditions by its appearance inside or outside loops and then only keep the ones appeared inside the loops in $\mathcal{B}$. We may also only keep the branch conditions which have some particular form, such as $a x \leqslant b$, etc.

The second method is different from the first one because it can be done in the pre-analysis or on the fly before splitting trees, thus no merging is needed during the analysis. This reduces the cost of the analysis, thus improves its 
efficiency. But because all the branch conditions being eliminated are not based on the information that is collected during the static analysis, the result may be less precise than the one generated from the first method. Moreover, eliminating branch conditions and merging their subtrees allow us to dynamically change the binary decision trees on the fly. This provides a more flexible way of adjusting the cost/precision ratio of the static analysis.

\section{Example}

Let us come back to Example 1. We choose the polyhedra abstract domain as the leaf abstract domain and we have $\mathcal{B}=\{\mathrm{x}<=50, \neg(\mathrm{x}<=50)\}$. Initially, we set $t=\left(\perp_{\ell} \mid\right)$ in the program point ${ }^{l}$. After the assignment " $\mathrm{x}=0 ; \mathrm{y}=0 ;$;, we have " $t=(\mathrm{x}=0 \wedge \mathrm{y}=0)$ ". Let $t_{i}$ be the abstract property at program point ${ }^{l}$ after the $i$-th iteration, then $t_{0}=(|\mathrm{x}=0 \wedge y=\mathrm{y}|)$. In the first iteration, we have to construct the binary decision tree when first reaching the branch test " $\mathrm{x}$ $<=50$ ". In this case, we have $\left.t_{0}^{\prime}=\llbracket \mathrm{x} \leq 50:(\mathrm{x}=0 \wedge \mathrm{y}=0),\left(\perp_{\ell}\right)\right) \rrbracket$. At the end of the first iteration, we get $t_{0}^{\prime \prime}=\llbracket \mathrm{x} \leq 50:(\mathrm{x}=1 \wedge \mathrm{y}=1),\left(\perp_{\ell}\right) \rrbracket$. Then $t_{1}=t_{0} \cup_{t} t_{0}^{\prime \prime}=\llbracket \mathrm{x} \leq 50:(\mid \mathrm{x}=\mathrm{y} \wedge 0 \leq \mathrm{x} \leq 1),\left(\left(\perp_{\ell}\right)\right)$. Afterwards, we apply the widening and get $t_{1}^{\prime}=t_{0} \nabla t_{1}=\llbracket \mathrm{x} \leq 50:(0 \leq \mathrm{x} \leq 50 \wedge \mathrm{x}=\mathrm{y} \mid),\left(0 \perp_{\ell} \mid\right) \rrbracket$. In the second iteration, the assignment " $\mathrm{x}++$;" leads to reconstruction on leaves, hence we get $t_{1}^{\prime \prime}=\llbracket \mathrm{x} \leq 50:(1 \leq \mathrm{x} \leq 50 \wedge \mathrm{x}=\mathrm{y} \mid),(\mid x=51 \wedge y=51) \rrbracket$. Then $t_{2}=t_{1} \cup_{t} t_{1}^{\prime \prime}=\llbracket \mathrm{x} \leq 50:(0 \leq \mathrm{x} \leq 50 \wedge \mathrm{x}=\mathrm{y}),((\mathrm{x}=51 \wedge \mathrm{y}=51) \rrbracket$. After the third iteration, $t_{3}=\llbracket \mathrm{x} \leq 50:(0 \leq \mathrm{x} \leq 50 \wedge \mathrm{x}=\mathrm{y} \mid),(\mathrm{x}+\mathrm{y}-102=$ $0 \wedge 51 \leq \mathrm{x} \leq 52 \mid) \rrbracket$. We then apply the widening and get $t_{3}^{\prime}=t_{2} \nabla t_{3}=\llbracket \mathrm{x} \leq 50$ : $(0 \leq \mathrm{x} \leq 50 \wedge \mathrm{x}=\mathrm{y}),(\mathrm{x}+\mathrm{y}-102=0 \wedge \mathrm{x} \geq 51))$. One more iteration yields $\left.t_{4}=\llbracket \mathrm{x} \leq 50:(0 \leq \mathrm{x} \leq 50 \wedge \mathrm{x}=\mathrm{y}),(\mathrm{x}+\mathrm{y}-102=0 \wedge 51 \leq \mathrm{x} \leq 103)\right)$. It follows that the program analysis converges. Hence $t_{4}$ is the invariant at program point ${ }^{l}$.

\section{Related Work}

A systematic characterization of the least bases for the disjunctive completion of abstract domains can be found in [8]. The trace partitioning using control flows was first introduced in [3]. A static analysis framework via trace partitioning was proposed by [11]. In this framework, the control flow is used to choose which disjunctions to keep but it lacks the merge of partitions, which may lead to exponential cost. In [13], a trace partitioning domain, where the partitioning of traces are based on the history of the control flow, has been proposed. The main difference between their partitionings and ours is we only use (part of) branch conditions while they are considering all conditions and other information.

Decision trees have been used for the disjunctive refinement of an abstract domain such as [10] for the interval abstract domain based on decision trees. A general segmented decision tree abstract domain, where disjunctions are determined by values of variables is introduced in [7]. Moreover, [16] proposed a general disjunctive refinement of an abstract domain based on decision 
trees extended with linear constraints for program termination. The difference between those works and ours is their partitionings are mainly based on the value of some variables while ours are directly based on the branch conditions.

There also exist several works on directly allowing disjunction in the domain, i.e., powerset domain [1]. In [15], the disjunctions are computed on an elaboration, which can be viewed as a multiply duplication, of the programs CFG structure. Moreover, our binary decision tree abstract domain functor can also be useful to scale traditional path-sensitive program analysis [17].

\section{Conclusion}

In this paper, we have introduced a series of abstractions which generates a set of branch condition paths. Those branch condition paths define a kind of trace partitioning on the concrete level (trace semantics of program). By using such information for trace partitioning, we proposed a binary decision tree abstract domain functor that allows finite disjunction of abstract properties generated by existing abstract domains ${ }^{1}$. We also discussed the implementation of our binary decision tree abstract domain functor by providing algorithms for inclusion test, meet and join, transfer functions and extrapolation operators. Although we bound the number of disjunctions only to the number of branch conditions in the program, the cost of our domain may still be excessive. Thus we also discussed how to limit the number of disjunctions. Our binary decision tree abstract domain functor may provide a flexible way of adjusting the cost/precision ratio for static analysis.

\section{References}

1. Bagnara, R., Hill, P.M., Zaffanella, E.: Widening operators for powerset domains. STTT 9(3-4), 413-414 (2007)

2. Chen, J.: SMT-based and disjunctive relational abstract domains for static analysis. Ph.D. thesis, New York University (May 2015)

3. Cousot, P.: Semantic foundations of program analysis. In: Muchnick, S., Jones, N. (eds.) Program Flow Analysis: Theory and Applications, pp. 303-342. PrenticeHall Inc., Englewood Cliffs (1981). Chapter. 10

4. Cousot, P., Cousot, R.: Static determination of dynamic properties of programs. In: Proceedings of the Second International Symposium on Programming, pp. 106-130. Dunod, Paris, France (1976)

5. Cousot, P., Cousot, R.: Abstract interpretation frameworks. J. Logic. Comput. 2(4), 511-547 (1992)

6. Cousot, P., Halbwachs, N.: Automatic discovery of linear restraints among variables of a program. In: Conference Record of the Fifth Annual ACM SIGPLAN-SIGACT Symposium on Principles of Programming Languages, pp. 84-97. ACM Press, New York, NY, Tucson, Arizona (1978)

\footnotetext{
${ }^{1}$ Note that all examples in this paper are numerical, but this does not mean that our abstract domain functor is limited to numerical abstract domains. Symbolic abstract domains are also allowed.
} 
7. Cousot, P., Cousot, R., Mauborgne, L.: A scalable segmented decision tree abstract domain. In: Manna, Z., Peled, D.A. (eds.) Time for Verification. LNCS, vol. 6200, pp. 72-95. Springer, Heidelberg (2010)

8. Giacobazzi, R., Ranzato, F.: Optimal domains for disjunctive abstract intepretation. Sci. Comput. Program. 32(1-3), 177-210 (1998)

9. Gopan, D., Reps, T.: Guided static analysis. In: Riis Nielson, H., Filé, G. (eds.) SAS 2007. LNCS, vol. 4634, pp. 349-365. Springer, Heidelberg (2007)

10. Gurfinkel, A., Chaki, S.: Boxes: a symbolic abstract domain of boxes. In: Cousot, R., Martel, M. (eds.) SAS 2010. LNCS, vol. 6337, pp. 287-303. Springer, Heidelberg (2010)

11. Handjieva, M., Tzolovski, S.: Refining static analyses by trace-based partitioning using control flow. In: Levi, G. (ed.) SAS 1998. LNCS, vol. 1503, pp. 200-214. Springer, Heidelberg (1998)

12. Jeannet, B., Miné, A.: Apron: a library of numerical abstract domains for static analysis. In: Bouajjani, A., Maler, O. (eds.) CAV 2009. LNCS, vol. 5643, pp. 661-667. Springer, Heidelberg (2009)

13. Mauborgne, L., Rival, X.: Trace partitioning in abstract interpretation based static analyzers. In: Sagiv, M. (ed.) ESOP 2005. LNCS, vol. 3444, pp. 5-20. Springer, Heidelberg (2005)

14. Miné, A.: The octagon abstract domain. High. Ord. Symbolic Comput. (HOSC) 19(1), 31-100 (2006). http://www.di.ens.fr/ mine/publi/article-mine-HOSC06.pdf

15. Sankaranarayanan, S., Ivančić, F., Shlyakhter, I., Gupta, A.: Static analysis in disjunctive numerical domains. In: Yi, K. (ed.) SAS 2006. LNCS, vol. 4134, pp. 3-17. Springer, Heidelberg (2006)

16. Urban, C., Miné, A.: A decision tree abstract domain for proving conditional termination. In: Müller-Olm, M., Seidl, H. (eds.) SAS 2014. LNCS, vol. 8723, pp. 302-318. Springer, Heidelberg (2014)

17. Winter, K., Zhang, C., Hayes, I.J., Keynes, N., Cifuentes, C., Li, L.: Path-sensitive data flow analysis simplified. In: Groves, L., Sun, J. (eds.) ICFEM 2013. LNCS, vol. 8144, pp. 415-430. Springer, Heidelberg (2013) 
15. Urban, C., Miné, A.: A decision tree abstract domain for proving conditional termination. In: Müller-Olm, M., Seidl, H. (eds.) Static Analysis - 21st International Symposium, SAS 2014, Munich, Germany, September 11-13, 2014. Proceedings. Lecture Notes in Computer Science, vol. 8723, pp. 302-318. Springer (2014)

16. Winter, K., Zhang, C., Hayes, I.J., Keynes, N., Cifuentes, C., Li, L.: Path-sensitive data flow analysis simplified. In: Groves, L., Sun, J. (eds.) Formal Methods and Software Engineering - 15th International Conference on Formal Engineering Methods, ICFEM 2013, Queenstown, New Zealand, October 29 - November 1, 2013,

Proceedings. Lecture Notes in Computer Science, vol. 8144, pp. 415-430. Springer (2013)

\section{A Trace Semantics}

Let $\Pi$ denote a set of traces. We define the trace semantics $\mathcal{S}^{t} \llbracket \mathrm{C} \rrbracket$ of commands $\mathrm{C}$ by induction on the syntactic structure of the commands:

$$
\begin{aligned}
& \mathcal{S}^{t} \llbracket \text { skip } \rrbracket \quad \triangleq\{\langle\text { skip, } \rho\rangle \stackrel{\text { skip }}{\longrightarrow}\langle\text { stop, } \rho\rangle \mid \rho \in \mathcal{E}\} \\
& \mathcal{S}^{t} \llbracket \mathrm{x}=\mathrm{E} \rrbracket \triangleq\{\langle\text { skip, } \rho\rangle \stackrel{\mathrm{x}=\mathrm{E}}{\longrightarrow}\langle\text { stop, } \rho[\mathrm{x}:=v]\rangle \mid \rho \in \mathcal{E} \wedge v \in \mathcal{E} \llbracket \mathrm{E} \rrbracket \rho\} \\
& \mathcal{S}^{t} \llbracket \mathrm{C}_{1} ; \mathrm{C}_{2} \rrbracket \triangleq\left\{\left(\pi ; C_{2}\right) \stackrel{\mathrm{A}}{\longrightarrow}\left\langle\mathrm{C}_{2}, \rho\right\rangle \stackrel{\mathrm{A}^{\prime}}{\longrightarrow} \pi^{\prime} \mid \rho \in \mathcal{E} \wedge\right. \\
& \left.\pi \stackrel{\mathrm{A}}{\rightarrow}\langle\text { stop, } \rho\rangle \in \mathcal{S}^{t} \llbracket \mathrm{C}_{1} \rrbracket \wedge\left\langle\mathrm{C}_{2}, \rho\right\rangle \stackrel{\mathrm{A}^{\prime}}{\longrightarrow} \pi^{\prime} \in \mathcal{S}^{t} \llbracket \mathrm{C}_{2} \rrbracket\right\} \\
& \mathcal{S}^{t} \llbracket \text { if (B) }\left\{\mathrm{C}_{1}\right\} \text { else }\left\{\mathrm{C}_{2}\right\} \rrbracket \triangleq \\
& \left\{\left\langle\text { if }(\mathrm{B})\left\{\mathrm{C}_{1}\right\} \text { else }\left\{\mathrm{C}_{2}\right\}, \rho\right\rangle \stackrel{\mathrm{B}}{\rightarrow}\left\langle\mathrm{C}_{1}, \rho\right\rangle \stackrel{\mathrm{A}}{\rightarrow} \pi \mid\right. \\
& \left.\rho \in \mathcal{E} \wedge \text { true } \in \mathcal{E} \llbracket \mathrm{B} \rrbracket \rho \wedge\left\langle\mathrm{C}_{1}, \rho\right\rangle \stackrel{\mathrm{A}}{\rightarrow} \pi \in \mathcal{S}^{t} \llbracket \mathrm{C}_{1} \rrbracket\right\} \\
& \cup\left\{\left\langle\text { if (B) }\left\{\mathrm{C}_{1}\right\} \text { else }\left\{\mathrm{C}_{2}\right\}, \rho\right\rangle \stackrel{\neg \mathrm{B}}{\longrightarrow}\left\langle\mathrm{C}_{2}, \rho\right\rangle \stackrel{\mathrm{A}}{\rightarrow} \pi \mid\right. \\
& \left.\rho \in \mathcal{E} \wedge \text { false } \in \mathcal{E} \llbracket \mathrm{B} \rrbracket \rho \wedge\left\langle\mathrm{C}_{2}, \rho\right\rangle \stackrel{\mathrm{A}}{\rightarrow} \pi \in \mathcal{S}^{t} \llbracket \mathrm{C}_{2} \rrbracket\right\} \\
& \mathcal{F}^{t i} \llbracket \text { while (B) }\{\mathrm{C}\} \rrbracket X \triangleq\{\langle\text { while (B) }\{\mathrm{C}\}, \rho\rangle \mid \rho \in \mathcal{E}\} \cup \\
& \left\{\pi \stackrel{\mathrm{A}}{\rightarrow}\langle\text { while }(\mathrm{B})\{\mathrm{C}\}, \rho\rangle \stackrel{\mathrm{B}}{\rightarrow}\left(\langle\mathrm{C}, \rho\rangle \stackrel{\mathrm{A}^{\prime}}{\longrightarrow} \pi^{\prime} \stackrel{\mathrm{A}^{\prime \prime}}{\longrightarrow}\left\langle\text { stop, } \rho^{\prime}\right\rangle\right) \text {; while (B) }\{\mathrm{C}\} \mid\right. \\
& \pi, \pi^{\prime} \in \Pi \wedge \pi \stackrel{\mathrm{A}}{\rightarrow}\langle\text { while }(\mathrm{B})\{\mathrm{C}\}, \rho\rangle \in X \wedge \text { true } \in \mathcal{E} \llbracket \mathrm{B} \rrbracket \rho \wedge \\
& \left.\left(\langle\mathrm{C}, \rho\rangle \stackrel{\mathrm{A}^{\prime}}{\longrightarrow} \pi^{\prime} \stackrel{\mathrm{A}^{\prime \prime}}{\longrightarrow}\left\langle\text { stop, } \rho^{\prime}\right\rangle\right) \in \mathcal{S}^{t} \llbracket \mathrm{C} \rrbracket\right\} \\
& \mathcal{S}^{t i} \llbracket \text { while (B) }\{\mathrm{C}\} \rrbracket \triangleq \operatorname{lfp} \subseteq \mathcal{F}^{t i} \llbracket \text { while (B) }\{\mathrm{C}\} \rrbracket \\
& \mathcal{S}^{t} \llbracket \text { while (B) }\{\mathrm{C}\} \rrbracket \triangleq\{\pi \stackrel{\mathrm{A}}{\rightarrow}\langle\text { while }(\mathrm{B})\{\mathrm{C}\}, \rho\rangle \stackrel{\neg \mathrm{B}}{\longrightarrow}\langle\text { stop, } \rho\rangle \mid \\
& \left.\pi \in \Pi \wedge \pi \stackrel{\mathrm{A}}{\rightarrow}\langle\text { while (B) }\{\mathrm{C}\}, \rho\rangle \in \mathcal{S}^{t i} \llbracket \text { while }(\mathrm{B})\{\mathrm{C}\} \rrbracket \wedge \text { false } \in \mathcal{E} \llbracket \mathrm{B} \rrbracket \rho\right\}
\end{aligned}
$$

\section{B Proof for Theorem 1}

The proof is by structural induction on the syntax of the command C.

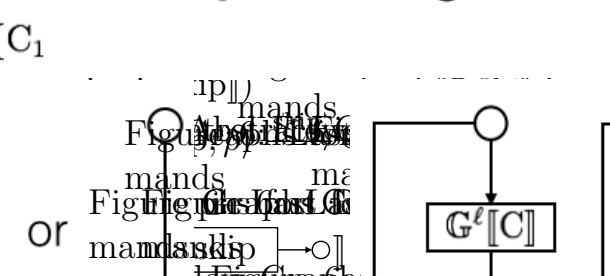

2definition of $\mathcal{S}^{t} \llbracket$ skip $\left.\rrbracket\right\}$ 2definition of $\alpha^{a}$ S 2definition of $\mathcal{G}^{a} \llbracket \circ \longrightarrow$ skip $\longrightarrow$ ○ा 


$$
\begin{aligned}
& \text { - } \text { Assignment } \\
& \alpha^{a}\left(\mathcal{S}^{t} \llbracket \mathrm{x}=\mathrm{E} \rrbracket\right) \\
&= \alpha^{a}(\{\langle\text { skip, } \rho\rangle \stackrel{\mathrm{x}=\mathrm{E}}{\longrightarrow}\langle\text { stop, } \rho[\mathrm{x}:=v]\rangle \mid \rho \in \mathcal{E} \wedge v \in \mathcal{E} \llbracket \mathrm{E} \rrbracket \rho\}) \\
&=\{\mathrm{x}=\mathrm{E}\} \\
& \triangleq \mathcal{G}^{a} \llbracket \mathrm{O} \longrightarrow \mathrm{x}:=\mathrm{E} \rightarrow \mathrm{O} \rrbracket \\
& \text { 2definition of } \mathcal{S}^{t} \llbracket \mathrm{x}=\mathrm{E} \rrbracket S \\
&\text { 2definition of } \left.\alpha^{a}\right\}
\end{aligned}
$$

\section{- Conditional}

$\alpha^{a}\left(\mathcal{S}^{t} \llbracket\right.$ if (B) $\left\{\mathrm{C}_{1}\right\}$ else $\left.\left\{\mathrm{C}_{2}\right\} \rrbracket\right)$

$=\alpha^{a}\left(\left\{\left\langle\right.\right.\right.$ if $(\mathrm{B})\left\{\mathrm{C}_{1}\right\}$ else $\left.\left\{\mathrm{C}_{2}\right\}, \rho\right\rangle \stackrel{\mathrm{B}}{\rightarrow}\left\langle\mathrm{C}_{1}, \rho\right\rangle \stackrel{\mathrm{A}}{\rightarrow} \pi \mid \rho \in \mathcal{E} \wedge$ true $\in \mathcal{E} \llbracket \mathrm{B} \rrbracket \rho \wedge$ $\left.\left\langle\mathrm{C}_{1}, \rho\right\rangle \stackrel{\mathrm{A}}{\rightarrow} \pi \in \mathcal{S}^{t} \llbracket \mathrm{C}_{1} \rrbracket\right\} \cup\left\{\left\langle\right.\right.$ if (B) $\left\{\mathrm{C}_{1}\right\}$ else $\left.\left\{\mathrm{C}_{2}\right\}, \rho\right\rangle \stackrel{\neg \mathrm{B}}{\longrightarrow}\left\langle\mathrm{C}_{2}, \rho\right\rangle \stackrel{\mathrm{A}}{\rightarrow} \pi \mid \rho \in$ $\mathcal{E} \wedge$ false $\left.\left.\in \mathcal{E} \llbracket \mathrm{B} \rrbracket \rho \wedge\left\langle\mathrm{C}_{2}, \rho\right\rangle \stackrel{\mathrm{A}}{\rightarrow} \pi \in \mathcal{S}^{t} \llbracket \mathrm{C}_{2} \rrbracket\right\}\right)$ 2definition of $\mathcal{S}^{t} \llbracket$ if $(\mathrm{B})\left\{\mathrm{C}_{1}\right\}$ else $\left\{\mathrm{C}_{2}\right\} \rrbracket \mathcal{S}$

$=\alpha^{a}\left(\left\{\left\langle\right.\right.\right.$ if $(\mathrm{B})\left\{\mathrm{C}_{1}\right\}$ else $\left.\left\{\mathrm{C}_{2}\right\}, \rho\right\rangle \stackrel{\mathrm{B}}{\rightarrow}\left\langle\mathrm{C}_{1}, \rho\right\rangle \stackrel{\mathrm{A}}{\rightarrow} \pi \mid \rho \in \mathcal{E} \wedge$ true $\in \mathcal{E} \llbracket \mathrm{B} \rrbracket \rho \wedge$ $\left.\left.\left\langle\mathrm{C}_{1}, \rho\right\rangle \stackrel{\mathrm{A}}{\rightarrow} \pi \in \mathcal{S}^{t} \llbracket \mathrm{C}_{1} \rrbracket\right\}\right) \cup \alpha^{a}\left(\left\{\left\langle\right.\right.\right.$ if (B) $\left\{\mathrm{C}_{1}\right\}$ else $\left.\left\{\mathrm{C}_{2}\right\}, \rho\right\rangle \stackrel{\neg \mathrm{B}}{\longrightarrow}\left\langle\mathrm{C}_{2}, \rho\right\rangle \stackrel{\mathrm{A}}{\rightarrow} \pi \mid$ $\rho \in \mathcal{E} \wedge$ false $\left.\left.\in \mathcal{E} \llbracket \mathrm{B} \rrbracket \rho \wedge\left\langle\mathrm{C}_{2}, \rho\right\rangle \stackrel{\mathrm{A}}{\rightarrow} \pi \in \mathcal{S}^{t} \llbracket \mathrm{C}_{2} \rrbracket\right\}\right)$

$$
\left\{\text { since } \alpha^{a}\left(S \cup S^{\prime}\right)=\alpha^{a}(S) \cup \alpha^{a}\left(S^{\prime}\right)\right\}
$$

$\left.\subseteq\left\{\mathrm{B} \cdot \alpha^{a}\left(\left\langle\mathrm{C}_{1}, \rho\right\rangle \stackrel{\mathrm{A}}{\rightarrow} \pi \mid\left\langle\mathrm{C}_{1}, \rho\right\rangle \stackrel{\mathrm{A}}{\rightarrow} \pi \in \mathcal{S}^{t} \llbracket \mathrm{C}_{1} \rrbracket\right\}\right)\right\} \cup\left\{\neg \mathrm{B} \cdot \alpha^{a}\left(\left\langle\mathrm{C}_{2}, \rho\right\rangle \stackrel{\mathrm{A}}{\rightarrow} \pi \mid\right.\right.$ $\left.\left.\left.\left\langle\mathrm{C}_{2}, \rho\right\rangle \stackrel{\mathrm{A}}{\rightarrow} \pi \in \mathcal{S}^{t} \llbracket \mathrm{C}_{2} \rrbracket\right\}\right)\right\} \quad$ 2definition of $\alpha^{a}$ and ignoring the test 5 $\left.\left.\left.\left.=\{\mathrm{B}\} \cdot\left\{\alpha^{a}\left(\pi^{\prime}\right) \mid \pi^{\prime} \in \mathcal{S}^{t} \llbracket \mathrm{C}_{1} \rrbracket\right\}\right)\right\} \cup\{\neg \mathrm{B}\} \cdot\left\{\alpha^{a}\left(\pi^{\prime \prime}\right) \mid \pi^{\prime \prime} \in \mathcal{S}^{t} \llbracket \mathrm{C}_{2} \rrbracket\right\}\right)\right\}$

2by defining the set of action paths concatenation $S \cdot S^{\prime} \triangleq\left\{\varpi \cdot \varpi^{\prime} \mid \varpi \in\right.$ $\left.S \wedge \varpi^{\prime} \in S^{\prime}\right\}$, letting $\pi^{\prime}=\left\langle\mathrm{C}_{1}, \rho\right\rangle \stackrel{\mathrm{A}}{\rightarrow} \pi$, and $\pi^{\prime \prime}=\left\langle\mathrm{C}_{2}, \rho\right\rangle \stackrel{\mathrm{A}}{\rightarrow} \pi \rho$

$=\{\mathrm{B}\} \cdot \alpha^{a}\left(\mathcal{S}^{t} \llbracket \mathrm{C}_{1} \rrbracket\right) \cup\{\neg \mathrm{B}\} \cdot \alpha^{a}\left(\mathcal{S}^{t} \llbracket \mathrm{C}_{2} \rrbracket\right) \quad$ 2definition of $\alpha^{a}$ S

$\subseteq\{\mathrm{B}\} \cdot \mathcal{G}^{a} \llbracket \mathrm{O} \longrightarrow \mathrm{C}_{1} \longrightarrow \mathrm{\circ} \rrbracket \cup\{\neg \mathrm{B}\} \cdot \mathcal{G}^{a} \llbracket \circ \rightarrow \mathrm{C}_{2} \longrightarrow \mathrm{O} \rrbracket$

2by structural induction hypothesis and the set of action paths concatenation $\cdot$ is $\subseteq$-increasing in both of its arguments. $\int$
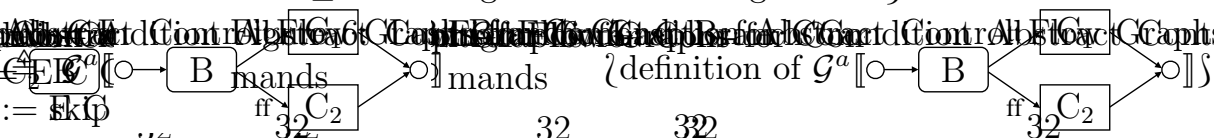

$$
\begin{aligned}
& \text { - Sequence } \\
& \alpha^{a}\left(\mathcal{S}^{t} \llbracket \mathrm{C}_{1} ; \mathrm{C}_{2} \rrbracket\right) \\
& =\alpha^{a}\left(\left\{\left(\pi ; C_{2}\right) \stackrel{\mathrm{A}}{\rightarrow}\left\langle\mathrm{C}_{2}, \rho\right\rangle \stackrel{\mathrm{A}^{\prime}}{\rightarrow} \pi^{\prime} \mid \pi \stackrel{\mathrm{A}}{\rightarrow}\langle\text { stop, } \rho\rangle \in \mathcal{S}^{t} \llbracket \mathrm{C}_{1} \rrbracket \wedge\left\langle\mathrm{C}_{2}, \rho\right\rangle \stackrel{\mathrm{A}^{\prime}}{\longrightarrow} \pi^{\prime} \in\right.\right. \\
& \left.\left.\mathcal{S}^{t} \llbracket \mathrm{C}_{2} \rrbracket\right\}\right) \quad \text { 2definition of } \mathcal{S}^{t} \llbracket \mathrm{C}_{1} ; \mathrm{C}_{2} \rrbracket \text { S } \\
& =\alpha^{a}\left(\left\{\pi \stackrel{\mathrm{A}}{\rightarrow}\langle\text { stop, } \rho\rangle \stackrel{\mathrm{A}^{\prime}}{\rightarrow} \pi^{\prime} \mid \pi \stackrel{\mathrm{A}}{\rightarrow}\langle\text { stop, } \rho\rangle \in \mathcal{S}^{t} \llbracket \mathrm{C}_{1} \rrbracket \wedge\left\langle\mathrm{C}_{2}, \rho\right\rangle \stackrel{\mathrm{A}^{\prime}}{\longrightarrow} \pi^{\prime} \in\right.\right. \\
& \left.\left.\mathcal{S}^{t} \llbracket \mathrm{C}_{2} \rrbracket\right\}\right) \quad \quad 2 \text { since } \alpha^{a}(\pi ; C)=\alpha^{a}(\pi) \mathcal{S} \\
& =\left\{\alpha^{a}(\pi) \cdot \mathrm{A} \cdot \mathrm{A}^{\prime} \cdot \alpha^{a}\left(\pi^{\prime}\right) \mid \pi \stackrel{\mathrm{A}}{\rightarrow}\langle\text { stop, } \rho\rangle \in \mathcal{S}^{t} \llbracket \mathrm{C}_{1} \rrbracket \wedge\left\langle\mathrm{C}_{2}, \rho\right\rangle \stackrel{\mathrm{A}^{\prime}}{\rightarrow} \pi^{\prime} \in \mathcal{S}^{t} \llbracket \mathrm{C}_{2} \rrbracket\right\} \\
& 2 \text { since } \alpha^{a}\left(\pi \stackrel{\mathrm{A}}{\rightarrow} \pi^{\prime}\right)=\alpha^{a}(\pi) \cdot \mathrm{A} \cdot \alpha^{a}\left(\pi^{\prime}\right) \oint \\
& =\left\{\alpha^{a}\left(\pi^{\prime \prime}\right) \cdot \alpha^{a}\left(\pi^{\prime \prime \prime}\right) \mid \alpha^{a}\left(\pi^{\prime \prime}\right) \in \mathcal{S}^{t} \llbracket \mathrm{C}_{1} \rrbracket \wedge \alpha^{a}\left(\pi^{\prime \prime \prime}\right) \in \mathcal{S}^{t} \llbracket \mathrm{C}_{2} \rrbracket\right\}
\end{aligned}
$$




$$
\begin{aligned}
& \text { 2letting } \left.\alpha^{a}\left(\pi^{\prime \prime}\right)=\pi \stackrel{\mathrm{A}}{\rightarrow}\langle\text { stop, } \rho\rangle \text { and } \alpha^{a}\left(\pi^{\prime \prime \prime}\right)=\left\langle\mathrm{C}_{2}, \rho\right\rangle \stackrel{\mathrm{A}^{\prime}}{\rightarrow} \pi^{\prime}\right\} \\
& =\alpha^{a}\left(\mathcal{S}^{t} \llbracket \mathrm{C}_{1} \rrbracket\right) \cdot \alpha^{a}\left(\mathcal{S}^{t} \llbracket \mathrm{C}_{2} \rrbracket\right)
\end{aligned}
$$

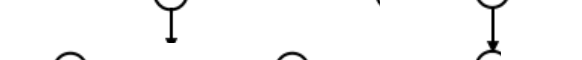

$$
\begin{aligned}
& \text { - Iteration, transformer } \\
& \alpha^{a}\left(\mathcal{F}^{t i} \llbracket \text { while (B) }\{\mathrm{C}\} \rrbracket(X)\right) \\
& =\alpha^{a}\left(\{ \langle \text { while } ( \mathrm { B } ) \{ \mathrm { C } \} , \rho \rangle | \rho \in \mathcal { E } \} \cup \left\{\pi \stackrel { \mathrm { A } } { \rightarrow } \langle \text { while } ( \mathrm { B } ) \{ \mathrm { C } \} , \rho \rangle \stackrel { \mathrm { B } } { \rightarrow } \left(\langle\mathrm{C}, \rho\rangle \stackrel{\mathrm{A}^{\prime}}{\rightarrow}\right.\right.\right. \\
& \pi^{\prime} \stackrel{\mathrm{A}^{\prime \prime}}{\longrightarrow}\left\langle\text { stop, } \rho^{\prime}\right\rangle \text { ) } \text { while (B) }\{\mathrm{C}\} \mid \pi, \pi^{\prime} \in \Pi \wedge \pi \stackrel{\mathrm{A}}{\rightarrow}\langle\text { while (B) }\{\mathrm{C}\}, \rho\rangle \in \\
& \left.\left.X \wedge \text { true } \in \mathcal{E} \llbracket \mathrm{B} \rrbracket \rho \wedge\left(\langle\mathrm{C}, \rho\rangle \stackrel{\mathrm{A}^{\prime}}{\longrightarrow} \pi^{\prime} \stackrel{\mathrm{A}^{\prime \prime}}{\longrightarrow}\left\langle\text { stop, } \rho^{\prime}\right\rangle\right) \in \mathcal{S}^{t} \llbracket \mathrm{C} \rrbracket\right\}\right) \\
& \text { 2definition of } \mathcal{F}^{t i} \llbracket \text { while (B) }\{\mathrm{C}\} \rrbracket S \\
& =\alpha^{a}(\{\langle\text { while }(\mathrm{B})\{\mathrm{C}\}, \rho\rangle \mid \rho \in \mathcal{E}\}) \cup \alpha^{a}\left(\left\{\pi \stackrel { \mathrm { A } } { \rightarrow } \langle \text { while } ( \mathrm { B } ) \{ \mathrm { C } \} , \rho \rangle \stackrel { \mathrm { B } } { \rightarrow } \left(\langle\mathrm{C}, \rho\rangle \stackrel{\mathrm{A}^{\prime}}{\longrightarrow}\right.\right.\right. \\
& \pi^{\prime} \stackrel{\mathrm{A}^{\prime \prime}}{\longrightarrow}\left\langle\text { stop, } \rho^{\prime}\right\rangle \text { ) } \text { while (B) }\{\mathrm{C}\} \mid \pi, \pi^{\prime} \in \Pi \wedge \pi \stackrel{\mathrm{A}}{\rightarrow}\langle\text { while (B) }\{\mathrm{C}\}, \rho\rangle \in \\
& \left.\left.X \wedge \text { true } \in \mathcal{E} \llbracket \mathrm{B} \rrbracket \rho \wedge\left(\langle\mathrm{C}, \rho\rangle \stackrel{\mathrm{A}^{\prime}}{\longrightarrow} \pi^{\prime} \stackrel{\mathrm{A}^{\prime \prime}}{\longrightarrow}\left\langle\text { stop, } \rho^{\prime}\right\rangle\right) \in \mathcal{S}^{t} \llbracket \mathrm{C} \rrbracket\right\}\right) \\
& \text { 2 since } \left.\alpha^{a}\left(S \cup S^{\prime}\right)=\alpha^{a}(S) \cup \alpha^{a}\left(S^{\prime}\right)\right\} \\
& \subseteq\{\varepsilon\} \cup\left\{\alpha ^ { a } \left(\pi \stackrel{\mathrm{A}}{\rightarrow}\langle\text { while }(\mathrm{B})\{\mathrm{C}\}, \rho\rangle \stackrel{\mathrm{B}}{\rightarrow}\left(\langle\mathrm{C}, \rho\rangle \stackrel{\mathrm{A}^{\prime}}{\rightarrow} \pi^{\prime} \stackrel{\mathrm{A}^{\prime \prime}}{\longrightarrow}\left\langle\text { stop, } \rho^{\prime}\right\rangle\right) ;\right.\right. \\
& \text { while (B) }\{\mathrm{C}\}) \mid \pi, \pi^{\prime} \in \Pi \wedge \pi \stackrel{\mathrm{A}}{\longrightarrow}\langle\text { while (B) }\{\mathrm{C}\}, \rho\rangle \in X \wedge\left(\langle\mathrm{C}, \rho\rangle \stackrel{\mathrm{A}^{\prime}}{\longrightarrow} \pi^{\prime} \stackrel{\mathrm{A}^{\prime \prime}}{\longrightarrow}\right. \\
& \left.\left.\left\langle\text { stop, } \rho^{\prime}\right\rangle\right) \in \mathcal{S}^{t} \llbracket \mathrm{C} \rrbracket\right\} \\
& \text { 2definition of } \alpha^{a} \text { and ignoring the result true } \in \mathcal{E} \llbracket \mathrm{B} \rrbracket \rho \text { of tests } \text { ) } \\
& =\{\varepsilon\} \cup\left\{\alpha^{a}(\pi \stackrel{\mathrm{A}}{\rightarrow}\langle\text { while }(\mathrm{B})\{\mathrm{C}\}, \rho\rangle) \cdot \mathrm{B} \cdot \alpha^{a}\left(\langle\mathrm{C}, \rho\rangle \stackrel{\mathrm{A}^{\prime}}{\rightarrow} \pi^{\prime} \stackrel{\mathrm{A}^{\prime \prime}}{\longrightarrow}\left\langle\text { stop, } \rho^{\prime}\right\rangle\right) \mid \pi, \pi^{\prime} \in\right. \\
& \left.\Pi \wedge \pi \stackrel{\mathrm{A}}{\rightarrow}\langle\text { while }(\mathrm{B})\{\mathrm{C}\}, \rho\rangle \in X \wedge\left(\langle\mathrm{C}, \rho\rangle \stackrel{\mathrm{A}^{\prime}}{\rightarrow} \pi^{\prime} \stackrel{\mathrm{A}^{\prime \prime}}{\longrightarrow}\left\langle\text { stop, } \rho^{\prime}\right\rangle\right) \in \mathcal{S}^{t} \llbracket \mathrm{C} \rrbracket\right\} \\
& 2 \text { since } \alpha^{a}\left(\pi \stackrel{\mathrm{A}}{\rightarrow} \pi^{\prime}\right)=\alpha^{a}(\pi) \cdot \mathrm{A} \cdot \alpha^{a}\left(\pi^{\prime}\right) \text { and } \alpha^{a}(\pi ; C)=\alpha^{a}(\pi) \rho \\
& =\{\varepsilon\} \cup\left\{\alpha^{a}\left(\pi^{\prime \prime}\right) \cdot \mathrm{B} \cdot \alpha^{a}\left(\pi^{\prime \prime \prime}\right) \mid \pi^{\prime \prime} \in X \wedge \pi^{\prime \prime \prime} \in \mathcal{S}^{t} \llbracket \mathrm{C} \rrbracket\right\} \\
& \text { 2letting } \pi^{\prime \prime}=\pi \stackrel{\mathrm{A}}{\longrightarrow}\langle\text { while }(\mathrm{B})\{\mathrm{C}\}, \rho\rangle \text { and } \pi^{\prime \prime \prime}=\langle\mathrm{C}, \rho\rangle \stackrel{\mathrm{A}^{\prime}}{\longrightarrow} \pi^{\prime} \stackrel{\mathrm{A}^{\prime \prime}}{\longrightarrow}\left\langle\text { stop, } \rho^{\prime}\right\rangle \rho \\
& =\{\varepsilon\} \cup \alpha^{a}(X) \cdot\{\mathrm{B}\} \cdot \alpha^{a}\left(\mathcal{S}^{t} \llbracket \mathrm{C} \rrbracket\right) \\
& \text { 2definition of } \alpha^{a} \text { and set of action paths concatenation\} } \\
& \subseteq\{\varepsilon\} \cup \alpha^{a}(X) \cdot\{\mathrm{B}\} \cdot \mathcal{G}^{a} \llbracket \circ \rightarrow \mathrm{C} \rightarrow \mathrm{O} \rrbracket
\end{aligned}
$$

2 by structural induction hypothesis and the set of action paths concatenation $\cdot$ is $\subseteq$-increasing in both of its arguments. $\int$

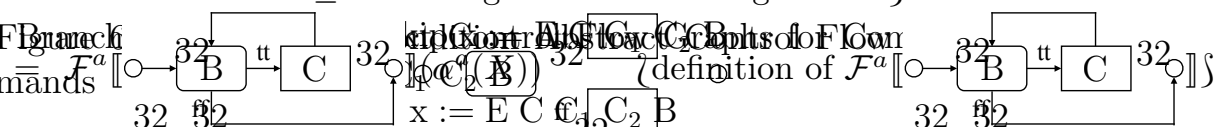

We have proved the semi-commutation property: 


$$
\begin{aligned}
& \forall X \in \wp(\Pi):
\end{aligned}
$$

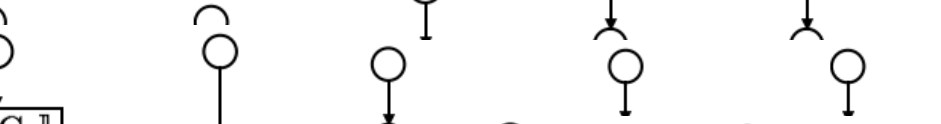

\section{- Union preservation}

Let us show that the iteration transformer preserves unions.

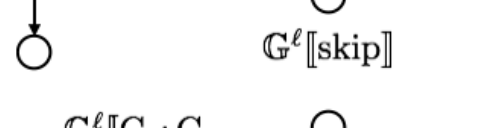

$$
\begin{aligned}
& =\{\varepsilon\} \cup\left(\bigcup_{i \in \Delta} S_{i}\right) \cdot\{\mathrm{B}\} \cdot \mathcal{G}^{a} \llbracket \circ \rightarrow \mathrm{C} \rightarrow \mathrm{C} \rrbracket \text { 2definition of } \mathcal{F}^{a} \llbracket \underbrace{\mathrm{B}}_{\mathrm{ff}}{ }^{\mathrm{tt}} \mathrm{C} \\
& =\{\varepsilon\} \cup \bigcup_{i \in \Delta}\left(S_{i} \cdot\{\mathrm{B}\} \cdot \mathcal{G}^{a} \llbracket \circ \longrightarrow \mathrm{C} \rightarrow \mathrm{O} \rrbracket\right) \\
& \text { 2since the concatenation - of sets of action paths preserves unions\} } \\
& =\bigcup_{i \in \Delta}\left(\{\varepsilon\} \cup S_{i} \cdot\{\mathrm{B}\} \cdot \mathcal{G}^{a} \llbracket \circ \rightarrow \mathrm{C} \rightarrow \mathrm{l}\right) \quad \text { (definition of } \cup \mathcal{S}
\end{aligned}
$$

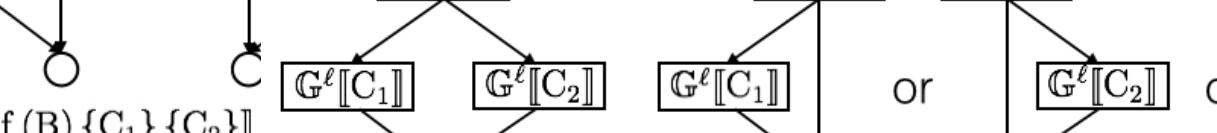

It follows that is $\subseteq$-increasing.

\section{- Fixpoint approximation}

A consequence of the semi-commutation property is that the abstraction of the concrete iterates $\left\langle S_{t}^{n}, n \in \mathbb{N}\right\rangle$ of $\mathcal{F}^{t i} \llbracket$ while (B) $\{\mathrm{C}\} \rrbracket$ from $\emptyset$ by $\alpha^{a}$ s overapproximated by the iterates $\left\langle S_{a}^{n}, n \in \mathbb{N}\right\rangle$ of $\mathcal{F}^{a} \llbracket \mathrm{B}$ stract: $\forall n \in \mathbb{N}: \alpha^{a}\left(S_{t}^{n}\right) \subseteq S_{a}^{n}$. The proof is by recurrence on $n \in \mathbb{N}$.

- $\alpha^{a}\left(S_{t}^{0}\right)=\alpha^{a}(\emptyset)=\emptyset=S_{a}^{0}$

- $\alpha^{a}\left(S_{t}^{n+1}\right)=\alpha^{a}\left(\mathcal{F}^{t i} \llbracket\right.$ while $\left.(\mathrm{B})\{\mathrm{C}\} \rrbracket\left(S_{t}^{n}\right)\right)$

2definition of iteration $\}$

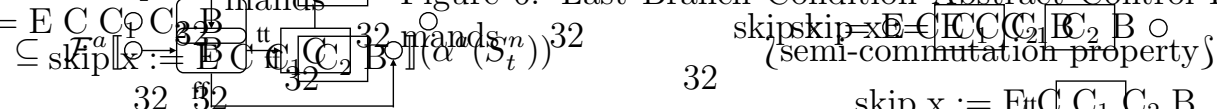

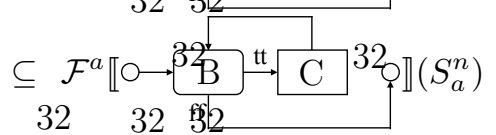

2by induction hypothesis $\alpha^{a}\left(S_{t}^{n}\right) \subseteq S_{a}^{n}$ and $\mathcal{F}^{a} \llbracket \mathrm{O} \rightarrow \stackrel{\mathrm{Bff}}{\mathrm{Bt}} \mathrm{C}$ @ $\rrbracket$ is $=S_{a}^{n+1} \subseteq$-increasing $\int$

2definition of iteration $\}$ 
It follows that we have the following fixpoint approximation,

$$
\begin{aligned}
& \alpha^{a}\left(\operatorname{lfp} \subseteq \mathcal{F}^{t i} \llbracket \text { while }(\mathrm{B})\{\mathrm{C}\} \rrbracket\right) \\
& \left.=\alpha^{a}\left(\bigcup_{n \in \mathbb{N}} S_{t}^{n}\right) \quad \text { 2since } \mathcal{F}^{t i} \llbracket \text { while }(\mathrm{B})\{\mathrm{C}\} \rrbracket \text { preserves unions }\right\} \\
& =\bigcup_{n \in \mathbb{N}} \alpha^{a}\left(S_{t}^{n}\right) \quad \text { 2since } \alpha^{a} \text { preserves unions } \int \\
& \subseteq \bigcup_{n \in \mathbb{N}}^{n \in \mathbb{N}} S_{a}^{n} \quad \text { 2since } \forall n \in \mathbb{N}: \alpha^{a}\left(S_{t}^{n}\right) \subseteq S_{a}^{n} \text { and definition of } \cup \text { S } \\
& =\operatorname{lfp}^{\subseteq} \mathcal{F}^{a} \llbracket \underbrace{\mathrm{B}}_{\mathrm{ff}} \stackrel{\mathrm{tt}}{\longrightarrow} \stackrel{\mathrm{C}}{\mathrm{O} \rrbracket}
\end{aligned}
$$

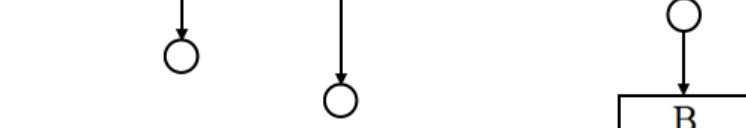

\section{- Iterates and least fixpoint of the iteration transformer}

Given a set $\mathcal{A}$ of action paths, let us define its natural powers as $\mathcal{A}^{0} \triangleq\{\varepsilon\}$, $\mathcal{A}^{1} \triangleq \mathcal{A}, \mathcal{A}^{n}=\underbrace{\mathcal{A} \cdot \ldots \cdot \mathcal{A}}_{n \text { times }}$ for $n>1$. The rule of powers $\mathcal{A}^{p+q}=\mathcal{A}^{p} \cdot \mathcal{A}^{q}$ is trivial.

Let us calculate the iterates $\left\langle F^{n}, n \in \mathbb{N}\right\rangle$ of $\mathcal{F}^{a} \llbracket \mathrm{ff}_{\mathrm{B}}^{\mathrm{B}} \mathrm{C}$. $F^{0}=\emptyset$

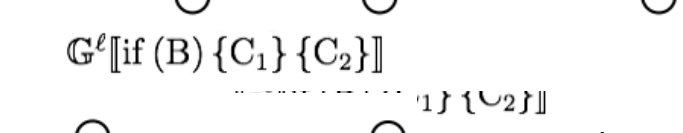

2definition of iteration, $\mathcal{F}^{a} \llbracket \underbrace{\mathrm{B}}_{\mathrm{ff}} \stackrel{\mathrm{C}}{\mathrm{tt}} \stackrel{\mathrm{C}}{\rightarrow}$ and $\forall \mathcal{A}: \emptyset \cdot \mathcal{A}=\mathcal{A}$ )

$-F^{2}=\mathcal{F}^{a} \llbracket \overbrace{\mathrm{ff}}^{\mathrm{B}} \stackrel{\mathrm{tt}}{\mathrm{O}} \mathrm{C}$

2definition of iteration and $\mathcal{F}^{a} \llbracket \underset{\mathrm{ff}}{\mathrm{B}} \stackrel{\mathrm{tt}}{\longrightarrow} \mathrm{C}$ oा)

$$
\left.=\{\varepsilon\} \cup\{\mathrm{B}\} \cdot \mathcal{G}^{a} \llbracket \mathrm{O} \rightarrow \mathrm{C} \rightarrow \mathrm{O} \rrbracket \quad \text { ince } \forall \mathcal{A}:\{\varepsilon\} \cdot \mathcal{A}=\mathcal{A}\right\}
$$

$=\left(\{\mathrm{B}\} \cdot \mathcal{G}^{a} \llbracket \circ \longrightarrow \mathrm{C} \rightarrow \mathrm{O} \rrbracket\right)^{0} \cup\left(\{\mathrm{B}\} \cdot \mathcal{G}^{a} \llbracket \circ \longrightarrow \mathrm{C} \rightarrow \mathrm{O} \rrbracket\right)^{1}$

2definition of natural powers $\mathcal{A}^{0} \triangleq\{\varepsilon\}$ and $\mathcal{A}^{1} \triangleq \mathcal{A}$ \}

$-F^{n}=\bigcup_{i=0}^{n-1}\left(\{\mathrm{~B}\} \cdot \mathcal{G}^{a} \llbracket \mathrm{O} \longrightarrow \mathrm{C} \longrightarrow \mathrm{O} \rrbracket\right)^{i} \quad$ 2induction hypothesis $\}$

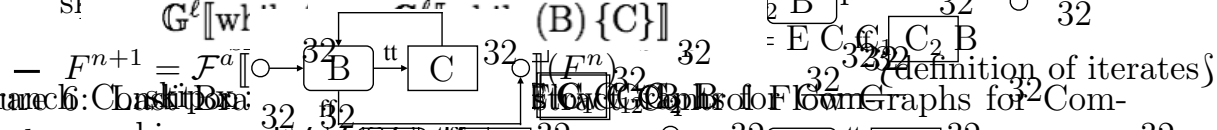




$$
\begin{aligned}
& =\{\varepsilon\} \cup\left(\bigcup_{i=0}^{n-1}\left(\{\mathrm{~B}\} \cdot \mathcal{G}^{a} \llbracket \circ \longrightarrow \mathrm{C} \rightarrow \mathrm{O} \rrbracket\right)^{i}\right) \cdot\{\mathrm{B}\} \cdot \mathcal{G}^{a} \llbracket \circ \longrightarrow \mathrm{C} \rightarrow \mathrm{O} \rrbracket
\end{aligned}
$$

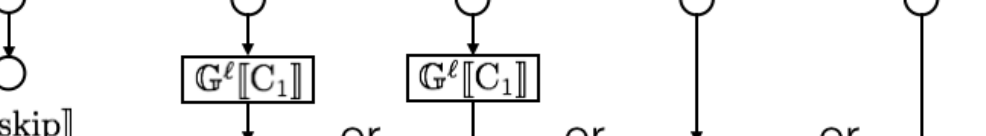

$$
\begin{aligned}
& =\left(\{\mathrm{B}\} \cdot \mathcal{G}^{a} \llbracket \mathrm{O}-\mathrm{C} \rightarrow \mathrm{O} \rrbracket\right)^{0} \cup \bigcup_{i=0}^{n-1}\left(\left(\{\mathrm{~B}\} \cdot \mathcal{G}^{a} \llbracket \mathrm{O} \rightarrow \mathrm{C} \rightarrow \mathrm{O} \rrbracket\right)^{i} \cdot\{\mathrm{B}\} \cdot\right. \\
& \left.\mathcal{G}^{a} \llbracket \mathrm{C} \longrightarrow \mathrm{C} \rightarrow \mathrm{O} \rrbracket\right) \\
& \text { 2definition of natural powers } \mathcal{A}^{0}=\{\varepsilon\} \text { and } \bigcup_{i \in \delta}\left(\mathcal{A}^{i}\right) \cdot \mathcal{A}=\bigcup_{i \in \delta}\left(\mathcal{A}^{i} \cdot \mathcal{A}\right) \mathcal{S} \\
& =\bigcup_{i=0}^{(n+1)-1}\left(\{\mathrm{~B}\} \cdot \mathcal{G}^{a} \llbracket \circ \rightarrow \mathrm{C} \rightarrow \mathrm{O} \rrbracket\right)^{i} \\
& \text { 2definition of natural powers } \mathcal{A}^{n+1}=\mathcal{A}^{n} \cdot \mathcal{A} \text { and definition of } \cup \mathcal{S} \\
& -\forall n \in \mathbb{N}: F^{n}=\bigcup_{i=0}^{n-1}\left(\{\mathrm{~B}\} \cdot \mathcal{G}^{a} \llbracket \mathrm{O}-\mathrm{C} \longrightarrow \mathrm{C} \rrbracket\right)^{i} \quad \text { 2by recurrence } S
\end{aligned}
$$

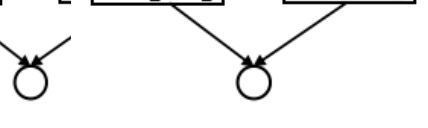

$$
\begin{aligned}
& =\bigcup_{n \in \mathbb{N}} F^{n} \quad \text { isince } \mathcal{F}^{a} \llbracket \underbrace{\mathrm{B}}_{\mathrm{ff}} \stackrel{\mathrm{Bt}}{\mathrm{C}} \mathrm{C} \rrbracket \text { preserves unions }\} \\
& =\bigcup_{n \in \mathbb{N}}\left(\{\mathrm{B}\} \cdot \mathcal{G}^{a} \llbracket \circ \rightarrow \mathrm{C} \rightarrow \circ \rrbracket\right)^{n} \quad \text { 2definition of natural powers } \mathcal{A}^{n} \text { and } \cup \mathcal{S}
\end{aligned}
$$

which we can write as $\left(\{\mathrm{B}\} \cdot \mathcal{G}^{a} \llbracket \circ \rightarrow \mathrm{C} \rightarrow \mathrm{\cap} \rrbracket\right)^{*}$.

$$
\begin{aligned}
& \text { - Iteration } \\
& \alpha^{a}\left(\mathcal{S}^{t} \llbracket \text { while (B) }\{\mathrm{C}\} \rrbracket\right) \\
& =\alpha^{a} \text { (let } \mathcal{S}^{t i} \llbracket \text { while }(\mathrm{B})\{\mathrm{C}\} \rrbracket \quad \triangleq \quad \operatorname{lfp} \subseteq \mathcal{F}^{t i} \llbracket \text { while }(\mathrm{B})\{\mathrm{C}\} \rrbracket \text { in }\{\pi \quad \mathrm{A} \quad \longrightarrow \\
& \langle\text { while (B) }\{\mathrm{C}\}, \rho\rangle \stackrel{\neg \mathrm{B}}{\longrightarrow}\langle\text { stop, } \rho\rangle \mid \pi \in \Pi \wedge \pi \stackrel{\mathrm{A}}{\rightarrow}\langle\text { while (B) }\{\mathrm{C}\}, \rho\rangle \in \\
& \left.\left.\mathcal{S}^{t i} \llbracket \text { while }(\mathrm{B})\{\mathrm{C}\} \rrbracket \wedge \text { false } \in \mathcal{E} \llbracket \mathrm{B} \rrbracket \rho\right\}\right) \\
& \text { 2definition of } \mathcal{S}^{t} \llbracket \text { while (B) }\{\mathrm{C}\} \rrbracket \int \\
& =\text { let } \mathcal{S}^{t i} \llbracket \text { while }(\mathrm{B})\{\mathrm{C}\} \rrbracket \quad \triangleq \quad \operatorname{lfp} \subseteq \mathcal{F}^{t i} \llbracket \text { while }(\mathrm{B})\{\mathrm{C}\} \rrbracket \text { in } \alpha^{a}(\{\pi \quad \stackrel{\mathrm{A}}{\rightarrow} \\
& \langle\text { while (B) }\{\mathrm{C}\}, \rho\rangle \stackrel{\neg \mathrm{B}}{\longrightarrow}\langle\text { stop, } \rho\rangle \mid \pi \in \Pi \wedge \pi \stackrel{\mathrm{A}}{\rightarrow}\langle\text { while (B) }\{\mathrm{C}\}, \rho\rangle \in \\
& \text { 2since } f(\text { let } \ldots \text { in } \ldots)=\text { let } \ldots \text { in } f(\ldots) S \\
& =\text { let } \mathcal{S}^{t i} \llbracket \text { while }(\mathrm{B})\{\mathrm{C}\} \rrbracket \triangleq \text { lfp } \subseteq \mathcal{F}^{t i} \llbracket \text { while }(\mathrm{B})\{\mathrm{C}\} \rrbracket \text { in } \alpha^{a}\left(\mathcal{S}^{t i} \llbracket \text { while }(\mathrm{B})\{\mathrm{C}\} \rrbracket\right) \text {. } \\
& \neg \mathrm{B} \\
& \text { 2definition of } \alpha^{a} \text { and ignoring the result false } \in \mathcal{E} \llbracket \mathrm{B} \rrbracket \rho \text { of test } \text { ) } \\
& =\alpha^{a}\left(\operatorname{lfp} \subseteq \mathcal{F}^{t i} \llbracket \text { while }(\mathrm{B})\{\mathrm{C}\} \rrbracket\right) \cdot\{\neg \mathrm{B}\} \quad \text { 2definition of let ... in ... }
\end{aligned}
$$




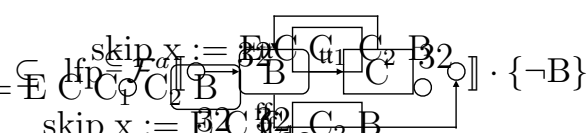

2by fixpoint approximation and $\cdot$ preserves unions so is $\subseteq$-increasing $\int$

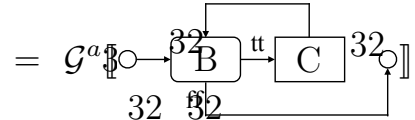

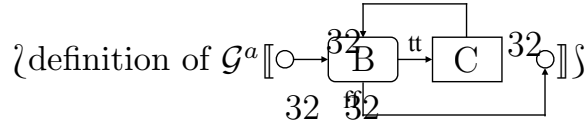

\section{Proofs in Section 3.2}

Theorem 2 (Homomorphic Abstraction). Given a function $h: C \mapsto A$, let $\alpha_{h}(X)=\{h(x) \mid x \in X\}$ and $\gamma_{h}(Y)=\{x \mid h(x) \in Y\}$, then $\alpha_{h}$ and $\gamma_{h}$ form $a$ Galois connection:

$$
(\wp(C), \subseteq) \underset{\alpha_{h}}{\stackrel{\gamma_{h}}{\leftrightarrows}}(\wp(A), \subseteq)
$$

Proof. For all $X \in \wp(C)$ and $Y \in \wp(A)$,

$$
\begin{array}{ll} 
& \alpha_{h}(X) \subseteq Y \\
\Longleftrightarrow \quad & \{h(x) \mid x \in X\} \subseteq Y \\
\Longleftrightarrow \quad & \forall x \in X: h(x) \in Y \\
\Longleftrightarrow & X \subseteq\{x \mid h(x) \in Y\} \\
\Longleftrightarrow & X \subseteq \gamma_{h}(Y)
\end{array}
$$

Proof of (1). By Theorem 2 where $h$ is $\alpha^{c}$.

Proof of (4). By Theorem 2 where $h$ is $\alpha^{d}$.

Proof of (7). By Theorem 2 where $h$ is $\alpha^{\ell}$.

Proof of (8). The composition of Galois connections is still a Galois connection. For all $\mathcal{A} \in \wp\left(\mathbb{A}^{*}\right)$ and $\mathcal{B} \in \wp\left(\left(\mathbb{A}^{B}\right)^{*} \backslash \mathbb{D}\right)$,

$$
\begin{aligned}
& \alpha^{b}(\mathcal{A}) \subseteq \mathcal{B} \\
& \Longleftrightarrow \quad \alpha^{\ell} \circ \alpha^{d} \circ \alpha^{c}(\mathcal{A}) \subseteq \mathcal{B} \\
& \Longleftrightarrow \quad \alpha^{d} \circ \alpha^{c}(\mathcal{A}) \subseteq \gamma^{\ell}(\mathcal{B}) \\
& \Longleftrightarrow \quad \alpha^{c}(\mathcal{A}) \subseteq \gamma^{d} \circ \gamma^{\ell}(\mathcal{B}) \\
& \Longleftrightarrow \mathcal{A} \subseteq \gamma^{c} \circ \gamma^{d} \circ \gamma^{\ell}(\mathcal{B}) \\
& \Longleftrightarrow \mathcal{A} \subseteq \gamma^{b}(\mathcal{B}) \\
& \text { 2definition of } \alpha^{b} \text { S } \\
& \text { 2 by }\left(\wp\left(\left(\mathbb{A}^{B}\right)^{*}\right), \subseteq\right) \underset{\alpha^{\ell}}{\stackrel{\gamma^{\ell}}{\leftrightarrows}}\left(\wp\left(\left(\mathbb{A}^{B}\right)^{*} \backslash \mathbb{D}\right), \subseteq\right) \int \\
& \left.2 \text { by }\left(\wp\left(\left(\mathbb{A}^{C}\right)^{*}\right), \subseteq\right) \underset{\alpha_{c}^{d}}{\stackrel{\gamma^{d}}{\leftrightarrows}}\left(\wp\left(\left(\mathbb{A}^{B}\right)^{*}\right), \subseteq\right)\right) \\
& 2 \text { by }\left(\wp\left(\mathbb{A}^{*}\right), \subseteq\right) \underset{\alpha^{c}}{\stackrel{\gamma^{c}}{\leftrightarrows}}\left(\wp\left(\left(\mathbb{A}^{C}\right)^{*}\right), \subseteq\right) \int \\
& \text { 2definition of } \gamma^{b} S
\end{aligned}
$$

